\title{
IV. Neue Bündnisse: Februar 1934 bis Oktober 1935
}

\section{Die Ereignisse des Februar 1934}

Die Schüsse am 6. Februar 1934 auf der Place de la Concorde schreckten die jungen Intellektuellen in ihren Redaktionsstuben auf. An dem Marsch auf das Palais Bourbon hatten sich die Anhänger des Ordre Nouveau und der Jeune Droite zwar nicht beteiligt, doch markierte dieser Tag für die Gruppierungen eine wichtige Zäsur. Das Ereignis veränderte nachhaltig das politische Klima in Frankreich und polarisierte die öffentliche Auseinandersetzung: Die Rechte sah darin den aufrichtigen Protest betrogener Bürger gegen die korrupte Regierung, für die Linke hingegen manifestierte sich darin der Versuch eines „faschistischen“ Umsturzes, dessen Drahtzieher die Führer der Ligen waren. In der Folge mobilisierten beide Lager ihre Anhänger, die mit Aufmärschen, Demonstrationen und Streiks die politische Agitation auf die Straße verlagerten.

Der Stavisky-Skandal, der den Aufmarsch ausgelöst hatte, war eine vergleichsweise geringfügige Affäre. Doch der mysteriöse Tod des betrügerischen Finanzberaters Stavisky verschärfte die Agitation gegen die regierenden Radikalen, von denen zahlreiche Abgeordnete in Geschäfte mit ihm verwickelt waren. Besonders die rechte Presse prangerte die Korruption und Handlungsunfähigkeit der Radikalen an und erneuerte ihre Attacken gegen die Republik. Die Agitation offenbarte das ganze Ausmaß der angestauten Unzufriedenheit. Sie wurde noch angefacht durch die Entlassung des Pariser Polizeichefs Chiappe, der den militanten Rechten nahestand und von der Linken für den Tod Staviskys verantwortlich gemacht wurde. Die Unruhen erreichten ihren Höhepunkt mit dem Protest von Hunderttausenden Bürgern, die dem Aufruf der Ligen folgten und am Abend des sechsten Februar gegen das Palais Bourbon, den Sitz der Kammer, vorrückten. Es kam zu Auseinandersetzungen mit Polizei und Sicherheitskräften, bei Schußwechseln wurden 14 Demonstranten getötet und etwa zweitausend verletzt. Der Führer der Feuerkreuzler, Oberst de La Rocque, blies im letzten Moment zum Rückzug und verhinderte damit den Sturm auf das Parlament. Als Reaktion auf dieses blutige Ende der Demonstration trat am nächsten Tag das Kabinett Daladier, von der bürgerlichen Presse als „Regierung der Mörder“ bezeichnet, zurück. Es war das erste Mal in der Geschichte der Dritten Republik, daß eine Regierung dem Druck der Straße hatte weichen müssen ${ }^{1}$.

Von der Linken wurde dieser Marsch auf das Palais Bourbon als Versuch eines „faschistischen" Staatsstreichs interpretiert und damit den Ligen ein organisierter Umsturzversuch unterstellt. Diese Sichtweise ist durch das Quellenmaterial weitgehend revidiert worden. Es gab keine Koordination unter den Anführern der Bewegungen, und die meisten Demonstranten trieb eher die Unzufriedenheit mit der Regierung als der Kampf gegen die Republik auf die Straße. Im Unterschied zu diesen Motiven aber boten die Programme der Ligen durchaus Anhaltspunkte für eine Orientierung, die sich nicht

${ }^{1}$ Vgl. Borne/Dubief, Crise, S. $104 \mathrm{ff}$. 
mehr eindeutig in die autoritär-nationalistische Tradition einreihen ließ, sondern die nach Ansicht einiger Historiker durchaus Merkmale faschistischer Bewegungen trug2.

Nach diesem Ereignis begann die Linke, ihre Kräfte unter dem Banner des „Antifaschismus" zu koordinieren. Der Anstoß ging von der Basis der Kommunisten, Sozialisten und Gewerkschaften aus. Streiks und Demonstrationen leiteten die Sammlungspolitik ein, die 1936 zum Sieg der Volksfront führen sollte. Dies war nur möglich durch ein Abrücken von der von der Komintern ausgegebenen Parole vom Kampf „Klasse gegen Klasse“. Erst durch die Preisgabe der "Sozialfaschismus-These" haben die französischen Kommunisten die Möglichkeit für die Zusammenarbeit mit den Sozialisten und bürgerlichen Linken geschaffen - eine Strategie, die auf dem VII. Weltkongreß der Komintern im Sommer 1935 von Dimitroff legitimiert wurde. Das öffnete den Weg für einen effektiven Aktionspakt der französischen Kommunisten mit den Sozialisten und bürgerlichen Linken zum Kampf gegen den "Faschismus"3.

Die Ereignisse hatten den Blick auf die Gefahr eines spezifisch französischen Faschismus gelenkt und diesem Thema eine breite Resonanz verschafft mit der Folge, daß die Unterscheidung von Freund und Feind sich beinahe ausschließlich über die Haltung zum "Faschismus" ergab und die politischen Auseinandersetzungen polarisierte. Seitdem verlagerte sich der Schwerpunkt des politischen Kampfes auf die Straße: die Aufmärsche der Ligen beantwortete die Linke mit Demonstrationen und Streiks, gegen die Bewegungen der extremen Rechten formierte sich in zunehmenden Maße eine organisierte Arbeiterschaft. Beide Seiten reagierten auf die Handlungsunfähigkeit der Regierung mit außerparlamentarischer Agitation.

\section{Isolation oder Aufbruch?}

Für Loubet del Bayle bildet der Februar 1934 die entscheidende Zäsur in der Geschichte der Nonkonformisten. Die Ereignisse auf der Place de la Concorde hätten abrupt das Ende der Zusammenarbeit zwischen den Nonkonformisten eingeleitet und den Willen zum Aufbruch in den Bahnen bestehender Bewegungen und Parteien kanalisiert. Daran ist richtig, daß sich die Beziehungen seitdem deutlich abkühlten. Um seine These zu untermauern, erwähnt er den Streit zwischen dem Ordre Nouveau und Esprit über den „Brief an Hitler“, die Abrechnung der Revue du siècle mit dem Titel „Nos adversaires et nos voisins" und die Nummer "Nous voulons" der Zeitschrift Ordre Nouveau4. Es stimmt, daß die erwähnten Artikel zur schärferen Akzentuierung der Differenzen beige-

2 Zu den Ereignissen vgl. die Quellensammlung: Le 6 février 1934, Hrsg. Berstein. Zur Faschismusdebatte: Soucy, Second Wave, S. 30ff.; ders., Das Wesen des Faschismus, S. 68ff.; ders., French Fascism and the Croix de Feu, S. 159-188; Irvine, Fascism in France, S. 271-295; Dobry, Février 1934, S. 511-533; Nolte, Die faschistischen Bewegungen, S. 290ff.; Sternhell, Ni droite, S. $25 \mathrm{ff}$. Die genannten Arbeiten richten sich insbesondere gegen den Ansatz der französischen Zeitgeschichtsschreibung, wie er von René Rémond und seinen Schülern vertreten wird.

${ }^{3}$ Jackson, Popular Front, S. 36ff.; Lefranc, Histoire du Front populaire, S. 13ff.; Wippermann, Faschismustheorien, S. $17 \mathrm{ff}$.

${ }^{4}$ Mounier, Prise de position, in: Esprit, H. 16, 1934, S. 533-540; ders., Réponse à l'Ordre Nouveau, in: Esprit, H. 19, 1934, S. 199-203; Revue du siècle, H. 10: „Nos adversaires et nos voisins“, 1934; ON, H. 9: „Nous voulons“, 1934, S. 1-32. 
tragen haben, die insbesondere die Gräben zwischen der Jeune Droite einerseits und Esprit und dem Ordre Nouveau andererseits vertieft haben, doch kam es zwischen keiner der drei Gruppierungen zum definitiven Bruch. Dem widerspricht schon der Befund, daß auch nach den Februarereignissen die wechselseitige Mitarbeit in den Zeitschriften fortgesetzt wurde 5 .

Das von ihm - in Anschluß an Emmanuel Mounier - gebrauchte Bild der Diaspora, in der sich die Gruppierungen seit Anfang 1934 befunden hätten, vermittelt einen falschen Eindruck ${ }^{6}$. Er folgt hier - wie die meisten französischen Historiker aus dem Umkreis des Institut d'Etudes Politiques - dem von Jean Touchard vorgegebenen Interpretationsrahmen, wonach der "Geist der dreißiger Jahre“ durch die Faschismusdebatte überlagert und von der metapolitischen Ebene in diejenige der politischen Parteikämpfe zurückgedrängt wurde ${ }^{7}$. Dieser Ansicht möchte ich entschieden widersprechen, und zwar in doppelter Hinsicht. Zunächst zum Kreis um Esprit: Wie die folgenden Kapitel deutlich machen, verfolgten deren Mitarbeiter weiterhin den Gedanken einer Revision überkommener Parteistandpunkte, wie sie in der Formel „weder rechts noch links“ ihren Ausdruck gefunden hat. Die Debatten lagen auch in der Folgezeit quer zu den politischen Auseinandersetzungen und lassen sich allenfalls vorübergehend auf die Konfrontation zwischen Volksfront- „Antifaschismus“ und Ligen- „Faschismus“ reduzieren.

Der Begriff der Diaspora suggeriert zudem, daß sich die Gruppierungen in die Isolation manövriert hätten. Dies ist aber mit Sicherheit nicht der Fall gewesen. Sie haben im Gegenteil seit 1934 ihre Beziehungen vervielfacht und somit ihre Anfangsphase als isolierte Intellektuellenzirkel überwunden. Sogar das Deutsche Nachrichtenbüro begann sich für die nonkonformistischen Kreise zu interessieren. In einem von ihm angeforderten Bericht der Deutschen Botschaft in Paris vom August 1934 rechnete man die Mitarbeiter von Ordre Nouveau, Esprit und der Revue du siècle zu den "geistig einflußreichen Gruppen“, deren Auftreten als ein „Gradmesser der geistigen Gärung“ gewertet wurde ${ }^{8}$. In der Tat ist die Phase seit Februar 1934 bestimmt durch eine mitunter hektische Aktivität bei der Suche nach neuen Verbündeten, bei der sich der Ordre Nouveau und die Jeune Droite in verschiedene Richtungen orientierten: Der Kreis der jungen und ehemaligen Maurrassianer näherte sich wieder verstärkt seinem Herkunftsmilieu in der extremen Rechten an, während der Ordre Nouveau nach einem kurzen „Flirt“ mit den Feuerkreuzlern eine Zusammenarbeit mit den Dissidenten aus dem linken Lager anvisierte.

${ }^{5}$ Nur einige Beispiele: Rougemont, Jeunesse déracinée, in: Revue du XX' siècle, H. 1, 1934, S. 1618; Fabrègues, XX' siècle, in: Esprit, H.21, 1934, S. 471-475; Maulnier, Les fautes de la droite, in: ON, H. 42, 1938, S. 2-6. Über die Fehlinterpretation des Verhältnisses Ordre Nouveau-Esprit vgl. Rougemont, Alexandre Marc, S. 51-69.

${ }^{6}$ Loubet del Bayle, Non-conformistes, S. 177.

7 Touchard, L'esprit des années trente, hier S. 108ff.; der Standpunkt Trebitschs unterscheidet sich allenfalls in Nuancen von dem Loubet del Bayles, vgl. Trebitsch, Le front commun, S. 221; die Marc-Biographie von Roy endet 1934. Diese Fixierung auf die frühen dreißiger Jahre kennzeichnet auch die Fabrègues-Biographie von Véronique Auzèpy-Chavagnac. In dieser Arbeit, die den Anspruch erhebt, die Zwischenkriegszeit zu behandeln, nimmt die entscheidende zweite Hälfte der dreißiger Jahre nicht einmal ein Zehntel der Darstellung ein; Auzèpy-Chavagnac, Fabrègues, hier S. 455-494.

8 PA/AA Botschaft Paris, 2144/4 Presseabteilung, 4. Jugendbewegung 1934-1938: Bericht über die französische Jugend vom August 1934. Auch Thomas S. Eliot begann sich für die Nonkonformisten zu interessieren: Eliot, A Commentary, in: The Criterion, April 1934, S. 451-454. 
Keineswegs jedoch fanden die Gruppierungen damit endgültig in ihre traditionellen politischen Lager zurück, vielmehr überkreuzten sich ihre Wege noch mehrfach. Die Sammlungsbestrebungen, zu denen der Cabier de Revendications einen ersten Auftakt bildete, wiederholten sich in unterschiedlichen Konstellationen.

„Vielleicht war dies das erste Mal, daß wir den Eindruck hatten, direkt von äußeren Ereignissen betroffen zu sein, die Konsequenzen zu erleben und sie ihrem Universum aus gedrucktem Papier zu entreißen", notierte Brasillach über die Ereignisse des Febru$\operatorname{ar}^{9}$. Deutlich läßt sich in den Äußerungen der Jeune Droite das Bemühen erkennen, die Toten der Place de la Concorde zu Märtyrern zu stilisieren. Für Jacques Reynaud waren sie von der demokratisch-freimaurerischen Staatsmacht heimtückisch niedergemetzelt worden. Der Aufmarsch, so der Mitherausgeber der Revue du Siècle, sei weit mehr als ein Zeichen der Unzufriedenheit mit der Regierung, er richte sich vielmehr grundsätzlich gegen den Parlamentarismus, gegen sechzig Jahre laizistischer und antinationaler Politik ${ }^{10}$. Jean de Fabrègues sah das Scheitern des Umsturzes in dem Mangel an präzisen Ideen begründet und rief die Anführer der Ligen dazu auf, den Blick auf die konstruktiven Erneuerungsversuche zu richten. Er bot ihnen an, seine Zeitschrift zum intellektuellen Zentrum eines neuen Umsturzes zu machen, und forderte sie zugleich auf, bis zur Entwicklung klarer Ziele die Agitation auf der Straße einzudämmen ${ }^{11}$.

Weniger zurückhaltend war der Kreis der ehemaligen Revue française. Als Antwort auf den gescheiterten Putsch hatten Robert Francis, Thierry Maulnier und Jean-Pierre Maxence ihr Werk Demain la France verfaßt, das sie den Toten des 6. Februar als den "ersten Zeugen der kommenden Revolution" widmeten ${ }^{12}$. Nie zuvor hatten sie sich so ausführlich mit begrifflichen Problemen wie Nation, Staat und Europa befaßt. Bisher hatten sie ihre Vorstellungen, ähnlich wie Maurras selbst, niemals als zusammenhängendes System präsentiert, wohl um damit dem Ideologievorwurf zu entgehen, den sie ihren Gegnern so gerne machten. Wenn sie nun von dieser Praxis abwichen, so möglicherweise deshalb, weil die Februarereignisse sie davon überzeugt hatten, daß es notwendig war, dem diffusen Protest in Zukunft klare Ziele voranzustellen.

Ungewöhnlich war auch der charismatische und aggressive Charakter des Nationalismus, der hier erstmals in aller Deutlichkeit zum Vorschein kam. Italien und Deutschland boten zwar Beispiele für die nationale Revolution, die die Jeune Droite in Frankreich anstrebte. Aber die Autoren beanspruchten für Frankreich den Rang des primus inter pares: die französische Nation besaß als historisch gewachsene Einheit eine höhere Legitimation als ihre Nachbarländer. „Frankreich ist der Grundtypus und das Modell aller Nationen. "13 Aus diesem Bewußtsein der Überlegenheit, gepaart mit einem zivilisatorischen Sendungsbewußtsein, folgerten die jungen Rechten das Recht und die Pflicht Frankreichs, seinen alten Führungsanspruch wieder zu erheben, um „die Rolle der Führer- und Leitnation, und um es genau zu sagen, der Hegemonialmacht “14 wieder einzu-

\footnotetext{
${ }_{9}$ Brasillach, Notre avant-guerre, in: ders., Euvres complètes, Bd. 6, S. 138.

${ }^{10}$ Reynaud, C'est une Révolution, in: Revue du siècle, H. 10, 1934, S. 2.

${ }^{11}$ Fabrègues, Nos adversaires et nos voisins, in: Revue du siècle, H. 10, 1934, S. 3; ders., L'Ordre Nouveau et nous, ebenda, S. 46-52, hier 46f.

12 Francis/Maulnier/Maxence, Demain la France, S. 1.

13 Ebenda, S. 102.

${ }^{14}$ Ebenda, S. 123.
} 
nehmen. Die jungen Revolutionäre verkündeten die Größe der französischen Nation mit einem ähnlichen Sendungsbewußtsein wie hundertfünfzig Jahre zuvor die Jakobiner, deren Ideologie sie radikal ablehnten. Ihnen warfen sie vor, zu Totengräbern des lebendigen französischen Nationalismus geworden zu sein. Die Französische Revolution hatte ihrer Ansicht nach mit dem Siegeszug der republikanischen Ideologie den Niedergang Frankreichs und Europas erst eingeleitet. Liberaler Kapitalismus und stalinistischer Totalitarismus hatten gleichermaßen ihren Ursprung im Geist von 1789. Die nationale Revolution mußte daher alle Formen dieser Ideologie beseitigen und an die Tradition des vorrevolutionären Nationalismus anknüpfen 15 .

Auch die Mitarbeiter des Ordre Nouveau erwiesen den Toten ihre Reverenz. Sie widmeten ihnen die Februarausgabe der Zeitschrift mit dem Thema „Französische Werte“: „In dem Moment, wo die Franzosen nach langer Zeit ihr Leben in einer Geste der Verzweiflung und des Abscheus geopfert haben, halten wir es für besonders dringend, sich auf die wahren französischen Werte zu besinnen. [...] Wir setzen unsere Arbeit fort und hoffen, daß sich künftig das Blutvergießen in improvisierten Vorläufern der notwendigen Revolution vermeiden läßt." ${ }^{16}$ Einen gewaltsamen Umsturz lehnten die Mitarbeiter des Ordre Nouveau entschieden ab. Für sie war der Blutzoll dieser Revolte vielmehr das Indiz für ihre mangelhafte Vorbereitung, die sie den Anführern des Aufstandes vorwarfen ${ }^{17}$. Nach Ansicht des Ordre Nouveau hatten die Chefs der Ligen in unverantwortlicher Weise das Leben ihrer Anhänger aufs Spiel gesetzt. Doch den Einsatz und die Opferbereitschaft der Demonstranten würdigten sie als aufrichtige Geste der Verzweiflung und der Auflehnung, der bisher lediglich die positiven Ziele fehlten. Diese versprach der Ordre Nouveau zu liefern.

Deutlicher als bisher mischten sich nationale Töne in die Debatte, wobei die Argumentation von dem Bemühen gekennzeichnet war, der "Weder-noch“-Position treu zu bleiben. In diesem Fall bedeutete dies die Ablehnung des jakobinischen und des integralen Nationalismus. Die von Aron und Dandieu in der Décadence skizzierte Theorie der „zwei Frankreich“ spitzten die Mitarbeiter in der Februarausgabe von Ordre Nouveau auf den Gegensatz von Staat und Nation zu: der offizielle, zentralistisch-bürokratische Machtstaat unterdrücke das "wirkliche" Frankreich der Vaterländer, das durch ein geistiges Band lebendiger Traditionen zusammengehalten werde. Andererseits waren die Feinde der Nation nicht wie bei der Action française die Einwanderer im rechtlichen oder ethnischen Sinne, sondern vielmehr alle diejenigen Ausländer und Franzosen, die eine "geistige Assimilation" verweigerten: die Nation wurde zu einer rein kulturellen Größe, was ihre Bedeutung keineswegs verringerte. Vielmehr trat an die Stelle des ethnischen Unterscheidungskriteriums ein sprachlich-ideologisches. Die Nation war das Ergebnis eines langen Zivilisationsprozesses, deren Erbe sich vor allem in der Sprache und Literatur manifestierte. Nach Ansicht Alexandre Marcs hatte jeder Franzose nicht nur das Recht, sondern die Pflicht, sich dies anzueignen, andernfalls blieb er ein Fremder.

\footnotetext{
${ }^{15}$ Ebenda, S. $128 \mathrm{ff}$.

${ }^{16}$ ON 8, Feb. 1934, S. 1.

17 Die Anwendung von physischer Gewalt hatten Aron und Dandieu schon in der Révolution nécessaire, S. IXf., abgelehnt.
} 
Die Größe der Nation garantierte dabei die Entfaltungsmöglichkeiten der Menschen: „Die Nation muß stark sein, damit der Mensch groß sein kann.“18

Deutlich wird in diesen Äußerungen das Bemühen um begriffliche Abgrenzung von den beiden großen Schulen des französischen Nationalismus: sowohl in der republikanischen Tradition als auch bei den Monarchisten der Action française sah man den Begriff der Nation zu machtstaatlichen Zwecken mißbraucht. Die Berechtigung der Existenz der Nation leitete der Ordre Nouveau aus der Bedeutung der französischen Kultur ab. Mit dem Hinweis auf die universale Gültigkeit dieser Kultur wurde der Führungsanspruch Frankreichs begründet. Robert Aron hat diese Vorstellung formuliert, indem er einen alten Topos auf charakteristische Weise abwandelte: „Jeder Mensch hat zwei Vaterländer, das seine und das des Personalismus, das wir aufs neue mit dem Boden und dem Vaterland Frankreich zur Übereinstimmung zu bringen versuchen." 19

\section{Antikapitalismus und Rassemblement-Idee}

Die Februarunruhen trafen das Land in einer Phase der langjährigen Depression. Um die wirtschaftliche Schwäche zu bekämpfen, betrieb man eine konsequente Deflationspolitik. Auch nach dem Februar 1934 versuchten die Regierungen, auf diese Weise die Wirtschaft anzukurbeln - doch ohne Erfolg: ein Wandel war bis zum Sieg der Volksfront nicht in Sicht ${ }^{20}$. Arbeitslosigkeit und Krise verschärften die sozialen Spannungen und drängten zu einem entschiedenen Wandel in der Wirtschafts- und Sozialpolitik, der jedoch paradoxerweise die politische Stabilität der Republik am meisten bedrohte.

Die Bildung der Volksfront läßt sich mit Hoffmanns Strukturmodell als ein Versuch beschreiben, die republikanische Synthese mit der Koalition der Linksparteien neu zu formieren, um auf diese Weise die Blockade der gesellschaftspolitischen Modernisierung aufzuheben und einen neuen Konsens zu erzielen. Doch die Sammlungsbewegung im Zeichen des "Antifaschismus" mobilisierte zugleich ihre Gegner auf der Linken und der Rechten und verschaffte den extremistischen Bewegungen erheblichen Zulauf ${ }^{21}$. Ebenso wie die Volksfront zielten auch alle anderen Sammlungsprogramme auf Versöhnung von Arbeitern, Bauern und Mittelschichten. Die antikapitalistische Programmatik hatte Konjunktur: bei der extremen Rechten verbreitete sich die Einsicht, daß die nationale Einheit nur durch die Überwindung der Klassengegensätze erreicht werden konnte. Seit 1934 läßt sich ein deutlicher Schub an sozialer Programmatik bei Ligen wie den Feuerkreuzlern und der Solidarité française wahrnehmen. Vielfach blieben diese Überlegungen jedoch mit den Vorstellungen eines hierarchischen Korporativsystems und paternalistischer Wohltätigkeit autoritären Denkmustern verhaftet ${ }^{22}$.

Insbesondere auf der Linken erhielt die Debatte neue Impulse durch den „Plan du Travail“" des belgischen Sozialisten Hendrik de Man, der die Überwindung des liberalen

${ }^{18}$ Marc, Tradition renouée, in: ON, H. 8, 1934, S. 1ff., Zitat S. 6.

${ }^{19}$ Aron, Liberté, in: ON, H. 8, 1934, S. 10.

${ }^{20}$ Kuisel, Capitalism, S. 93-96.

${ }^{21} \mathrm{Vgl}$. Hoffmann, Paradoxes, S. $26 \mathrm{ff}$.

22 Zur SF vgl. das Programm von Dez. 1935, abgedruckt bei Machefer, Ligues, S. 42-44, vgl. Soucy, Second Wave, S. 87ff., zu den Feuerkreuzlern ebenda, S. $175 \mathrm{ff}$. 
Kapitalismus versprach. In der Folge entwickelten auch der Dachverband der Gewerkschaften Confédération générale du travail (C.G.T.), die Neosozialisten und einige Intellektuellenzirkel eigene Pläne, mit denen sie alternative Sammlungsprogramme zur Volksfront vorstellten ${ }^{23}$.

Die wirtschaftspolitischen Vorstellungen der Jeune Droite waren von ihrer Herkunft aus dem Milieu der Action française geprägt und der Tradition des korporatistischen $\mathrm{Pa}$ ternalismus verpflichtet. Deutlich kam in den Verlautbarungen die Vorstellung zum Ausdruck, daß soziale Gerechtigkeit sich weniger durch ein klar umrissenes wirtschaftliches und soziales Reformprogramm als vielmehr durch die Verteidigung "naturgegebener" Ordnungen wie Familie und Nation erreichen ließ24. Der Kreis um die Revue $d u$ Siècle setzte sich für eine korporatistische Ordnung ein, die nichts anderes als eine Neuauflage der Ideen von René de La Tour du Pin war. Dessen hundertsten Geburtstag feierte die Zeitschrift 1934 in einer Sondernummer mit einem entschiedenen Bekenntnis zum paternalistischen Korporatismus - eine Haltung, die angesichts der engen personellen Verflechtung der Gruppierung mit dem mittelständischen Unternehmerverband der Union des Corporations Françaises (U.C.F.) und seinem intellektuellen Zentrum, dem "Cercle de La Tour du Pin“, nicht verwundern kann²5.

Die jungen Rechten sahen sich besonders dem Vorwurf ausgesetzt, gemeinsame Sache mit der wirtschaftsliberalen Rechten zu machen - ein Vorwurf, den sie mit Hinweis auf die Nähe zum faschistischen Korporativsystem zu entkräften versuchten. Beide, so betonte Jacques Saint-Germain, seien "zutiefst antikapitalistisch und revolutionär"26. Beide bekämpften seiner Ansicht nach wirkungsvoll jegliche Art von Finanzspekulation. Nur sie könnten das Recht des Menschen auf Arbeit verwirklichen, das in einer liberalen Wirtschaftsordnung den Kapitalinteressen geopfert werde. Einen grundsätzlichen Unterschied zwischen der Schule La Tour du Pins und dem faschistischen Korporatismus sah Saint-Germain nur in der Rolle des Staates: Einen direkten Einfluß des Staates auf die Genossenschaften lehnte er ab. Er legte vielmehr Wert auf den spontanen, freiwilligen Charakter der Organisation, in der der Staat nur die Rolle einer obersten Kontrollinstanz innehaben sollte. Die Verwaltung sollte weitgehend dezentralisiert werden. Das Genossenschaftsmodell wollte man auf alle Bereiche der Gesellschaft ausdehnen. Die Berufsstände sollten neben den wirtschaftlichen auch politisch-administrative

${ }^{23}$ Brender, Kollaboration, S. 43ff.; zu Bergerys Frontisme vgl. Burrin, Dérive, S. 117ff.; Kuisel, Capitalism, S. 98ff.; Biard, Le socialisme.

${ }^{24}$ Fabrègues, Nécessité de s'engager, in: Revue du XXe siècle, H. 1, 1934, S. 38.

${ }^{25}$ Pour le centenaire de la Tour du Pin, in: Revue du siècle, H. 11/12, 1934 mit Beiträgen unter anderem von Fabrègues, Magniez und Saint-Germain. Fabrègues selbst war Ende der zwanziger Jahre als Redner bei den Konferenzen des Verbandes aufgetreten und pflegte den Kontakt weiterhin in den dreißiger Jahren. Fabrègues, Maulnier und Roger Magniez hatten leitende Funktionen im „Cercle La Tour du Pin“ inne, dem ideologischen Zentrum des U.C.F.; Magniez schrieb zudem regelmäßig für das Verbandsorgan La Production française. Zu Fabrègues vgl. AN F7 13 983, Bericht vom 24. 5. 1936; Auzèpy-Chavagnac, Fabrègues, S. 210, 256ff., 324. Magniez war 1931 Präsident des Cercle La Tour du Pin, wie aus seinen regelmäßigen Beiträgen für La Production française hervorgeht, für die er zumindest bis 1934 regelmäßig schrieb, vgl. den Untersuchungsbericht vom März 1934, APP Ba 1893; zu Maulnier vgl. AN F7 13 983/14, Bericht vom Mai 1936.

${ }^{26}$ Saint-Germain, Vers la corporation, in: Revue du XXe siècle, H. 1, 1934, S. 50. 
Funktionen wahrnehmen. An die Stelle der gewählten Körperschaften in Stadt, Provinz und Parlament traten die berufsständischen Interessenvertretungen ${ }^{27}$.

Die gläubigen Katholiken unter den jungen Rechten bemühten sich, ihre Glaubensgenossen auf national-antirepublikanische Ziele einzuschwören. Schärfer als sonst griff Fabrègues all diejenigen Strömungen des Katholizismus an, die nicht in der gleichen radikalen Weise wie die Jeune Droite die Demokratie bekämpften und für die Ligen eintraten. Seine Angriffe richteten sich gegen Jacques Maritain ebenso wie gegen die Zeitschriften Esprit, die von den Jesuiten herausgegebene La vie intellectuelle und das Anfang 1934 von den Dominikanern in Juvisy gegründete Wochenblatt Sept. Die ablehnende Haltung dieser Sprachrohre des Katholizismus gegenüber den Ligen war für Fabrègues ein deutliches Zeichen für deren Mangel an engagiertem Patriotismus ${ }^{28}$.

Gleichzeitig verstärkte die Jeune Droite ihre Verbindungen zum Thronprätendenten, dem Grafen von Paris. Mit Beifall wurde dessen Initiative aufgenommen, mit dem Ende 1934 gegründeten Courrier Royal ein Sammelbecken der royalistischen Kräfte in Frankreich zu schaffen ${ }^{29}$. Die Initiative des Grafen war eine Reaktion auf den Mißerfolg des Februaraufstandes, für den er die Action française maßgeblich verantwortlich machte. Seine Zeitschrift sollte das verwirklichen, wozu sich die Bewegung von Maurras außerstande gezeigt hatte: die Aktivitäten aller Gruppierungen der extremen Rechten zu vereinigen. Getreu dem auf jeder Titelseite proklamierten Motto „die Monarchie ist keine Partei“ vermied der Courrier weitgehend polarisierende Polemik: Inhaltlich distanzierte sich der Graf von dem Mittelschichts-Elitenkult der Action française-Intellektuellen und appellierte ausdrücklich auch an Arbeiter und Bauern. Die Zeitschrift beteiligte sich nicht an den heftigen antisemitischen Kampagnen der extremen Rechten, zollte aber andererseits der Action française stets gebührenden Respekt ${ }^{30}$.

Es ist wahrscheinlich, daß ein großer Teil der Jeune Droite von Anfang an das Unternehmen des Grafen unterstützte. Seit Oktober 1935, als die Anonymität der Autoren nach und nach aufgehoben wurde, kann der Mitarbeiterkreis namentlich festgemacht werden: Maulnier, Fabrègues, Saint-Germain und Héritier hatten sich bald eigene Kolumnen erobert ${ }^{31}$. Das Blatt verzeichnete innerhalb kurzer Zeit einen beachtlichen Erfolg, konnte seit September 1935 sogar wöchentlich erscheinen und entwickelte sich bald zu einer ernsten Konkurrenz der Action française, die dies in einer deutlichen $\mathrm{Ab}$ wanderungsbewegung der Leser zu spüren bekam ${ }^{32}$.

${ }^{27}$ Saint-Germain, Vers la corporation, in: Revue du XXe siècle, H. 2, 1934, S. 57-61.

${ }^{28}$ Fabrègues, Où sont les catholiques français?, in: Revue du XXe siècle, H. 6, 1935, S. 4-9; eine noch schärfere Kritik an Esprit findet sich bei Maulnier, Note sur la revue "Esprit“, ebenda, S. 1012.

${ }^{29}$ Fabrègues/Francis/Maxence/Maulnier, Une politique vivante, in: Revue du XXe siècle, H. 3, 1935, S. 3-7.

30 Weber, Action Française, S. 405f.

31 Von den Anhängern der Jeune Droite arbeiteten folgende für den Courrier Royal: Jean Baudry, Jean de Fabrègues, Jean Héritier, Jean Loisy, Thierry Maulnier, Jean Saillenfest, Jacques SaintGermain, Emile Vaast. Dies hinderte jedoch Maulnier nicht daran, weiterhin regelmäßig für die Action française zu schreiben.

32 Darüber hinaus strich der Graf Anfang 1936 sämtliche finanzielle Unterstützung für die Action française, AN F7 12964, Bericht vom 11.3. 1936. 
Thierry Maulnier, Jean-Pierre Maxence und Robert Francis begnügten sich nicht mit diesen Absichtsbekundungen, sie engagierten sich vielmehr direkt in den Reihen einer Bewegung, über deren faschistischen Charakter sich die Historiker einig sind: der Solidarité Française, einer Liga, die ihren Willen zum Staatsstreich bei der Feier ihres zweijährigen Bestehens vollmundig mit dem Motto bekräftigte: „1789: Französische Revolu-

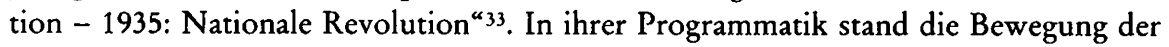
Action française sehr nahe: der Ruf nach einem starken Staat paarte sich auch hier mit Fremdenfeindlichkeit und Antisemitismus. Nach dem Tod ihres Mäzens François Coty 1934 machte sich die Liga unter Jean Renaud die Forderungen zu eigen, die die Action Française in früheren Zeiten einmal erhoben hatte: eine kompromißlose antiparlamentarische Haltung und den Willen zum Umsturz, den die Royalisten mit ihrer Annäherung an die parlamentarische Rechte aufgegeben hatten ${ }^{34}$. Zur Durchsetzung ihrer Ziele verfügte die Solidarité über eine paramilitärisch organisierte Kampftruppe, die besonders in der Pariser Region aktiv war. Ihre mit blauen Hemden uniformierten Anhänger rekrutierte sie vorwiegend aus dem kleinbürgerlichen Milieu ${ }^{35}$.

Ähnlich wie die übrigen Ligen profitierte die Bewegung vom Stavisky-Skandal und verzeichnete seit Anfang 1934 einen bedeutenden Zulauf. Ihre Größe ist in der älteren Forschung häufig stark unterschätzt worden. Die Pariser Sûreté bezifferte die Zahl ihrer Anhänger Mitte Februar auf 158000 bis 180000 - weitaus mehr, als die Feuerkreuzler zum gleichen Zeitpunkt hatten ${ }^{36}$.

In ihrem Werk Demain la France hatten sich die drei Autoren stark den Positionen angenähert, die auch die Solidarité vertrat. Das Buch liest sich wie ein Entwurf der Leitlinien, mit denen Jean Renaud 1935 die Abkehr vom reformistischen Kurs unter Coty

33 APP Ba 1960, Abschrift eines Plakates der Veranstaltung in der Salle Wagram am 6. 5. 1935. Als Redner trat neben Jean Renaud, Charles Vioud, Louis Mouilliseaux, Jacques Fromentin auch Maxence auf. Dies ist der erste Beleg von Maxences Aktivität in den Reihen der SF. Der genaue Zeitpunkt des Beitritts läßt sich weder bei ihm noch bei Maulnier und Francis bestimmen. Maulnier und Francis tauchten spätestens im Oktober bzw. November 1935 in den Reihen der SF auf, vgl. Aron, Vapeurs d'huile, in: Bulletin ON, Nr. 6, 15. 11. 1935, S. 2 (Maxence, Francis, Maulnier) und APP Ba 1960, Bericht vom 11.10. 1935 (Maulnier).

${ }^{34}$ Im Unterschied zu Coty, der seiner Taktik noch den Anschein von Legaliät gab, machte Renaud keinen Hehl aus seinen Absichten eines Staatsstreichs; Soucy, French Fascism. The Second Wave, S. 87.

35 Wenn es darum ging, Präsenz auf der Straße zu demonstrieren, mußten im Zweifelsfall auch ideologische Motive zurückstehen. Die SF rekrutierte nämlich auch im Milieu der maghrebinischen Einwanderer. Sie wurden mit Geld und Vergünstigungen bewogen, in den Reihen der Solidarité zu marschieren; Soucy, Second Wave, S. 59ff. Spätestens seit Mitte 1935 waren die Pariser Straßen beinahe täglich Schauplatz von Übergriffen der Bewegung gegen die jüdische Bevölkerung; APP Ba 1812, Briefe von Bernard Lecache, L.I.C.A., an den Polizeipräfekten vom 26.9. 1935 und 14. 9. 1938 sowie Bericht vom 23.9. 1938.

${ }^{36}$ AN F7 13 238, Bericht vom 12. 2. 1934, weitere 80000 waren mit ihren Beitragszahlungen in Verzug; vgl. Soucy, Second Wave, S. 63. Diese Zahlen müssen - wie bei allen übrigen Ligen auch - relativiert werden. Nur ein kleiner Teil von ihnen war auch wirklich aktiv. Serge Berstein schätzt die Zahl der SF-Truppen bei dem Aufmarsch am sechsten Februar auf 1500; Berstein, Le 6 février, S. 160. Rémond, Droites, S. 207 bezifferte die Gesamtzahl der Anhänger noch auf maximal 10000. 
und die Hinwendung zu einem genuin faschistischen Kurs proklamierte ${ }^{37}$. Der Staatschef sollte nach dem Willen von Francis, Maxence und Maulnier nahezu uneingeschränkte Vollmachten genießen und weitgehend unabhängig von demokratischen Legitimationsverfahren sein. Dabei war es den drei Autoren gleichgültig, wie sie ausdrücklich betonten, ob ein Prinz oder ein anderer Herrscher die Geschicke dieses Staates leitete. Es kam ihnen vielmehr darauf an, das parlamentarische Repräsentativsystem zu bekämpfen und dem Staatsoberhaupt völlige Unabhängigkeit von den Volksvertretern zu gewähren ${ }^{38}$. Damit sprachen sie zum erstenmal offen aus, was den Monarchisten aus dem Umkreis der Action française schon lange unterstellt wurde, nämlich daß es ihnen nicht so sehr um die Restauration der Monarchie ging, die Person des Thronprätendenten in Wahrheit nebensächlich war. Die gegenrevolutionäre Rhetorik war nicht wörtlich gemeint. Mit der „Restauration der Monarchie“ wollten sie keineswegs Königtum und Adel in ihre alten Rechte einsetzen und die erblichen Privilegien wiederherstellen. Anstelle einer landständischen forderten sie eine berufsständische Vertretung. Die Anknüpfung an die Verhältnisse vor 1789 war vor allem funktional zu verstehen: als Wendung gegen die Republik und Ruf nach autoritärer Herrschaft.

Getreu dem Leitsatz von Maurras, „Oben die Autorität, unten die Freiheiten“"39, setzten sich die Autoren für eine starke politische Zentralgewalt ein, die den Bürgern die "Last" der Politik abnehmen sollte. Darüber hinaus machten sie sich zu Anwälten der Dezentralisierung - ein altes Thema der Action française im Kampf gegen den republikanischen Zentralismus, das nach dem Weltkrieg weitgehend in Vergessenheit geraten war. Ähnliche Forderungen machte sich Jean Renaud in seinem Programm von $1935 \mathrm{zu}$ eigen. So versprach die Solidarité das zu erfüllen, was die Action française einmal gefordert hatte ${ }^{40}$.

Maulnier, Maxence und Francis plädierten für den Abbau der Bürokratie und für einen Rückzug des Staates aus der Wirtschafts- und Sozialpolitik. In diesen Bereichen wollten sie der regionalen und kommunalen Verwaltung weitgehende Freiheiten überlassen. Schiffahrt und Eisenbahn sollten privatisiert werden, der Post- und Telephondienst in private Hände übergehen, wobei dem Staat lediglich eine Kontrollfunktion zukommen sollte. Dem Entwurf zufolge übernahmen die Berufsgenossenschaften die Verantwortung für die Sozialversicherungen. Obendrein beabsichtigten sie auch, das Erziehungswesen dem staatlich-laizistischen Einfluß zu entziehen: Ein wirksameres Programm zum Abbau des Wohlfahrtsstaates ließ sich kaum entwerfen.

Vom Antikapitalismus, den sie so vehement proklamierten, bleibt bei näherer Betrachtung wenig Substanz. Die Kritik der drei Autoren richtete sich ausschließlich gegen das Finanzkapital, die „anonyme“ Macht der Banken und der Aktiengesellschaften, während das Privateigentum unantastbar blieb und der Grundbesitz geradezu als Voraussetzung einer "nicht-entfremdeten" Existenz galt. Sie versprachen, die Konzentration der Wirtschafts- und Finanzmacht zu bekämpfen und sich für die Belange der Bauern, Arbeiter sowie des kleinen und mittleren Bürgertums einzusetzen. „In Zukunft

\footnotetext{
${ }^{37}$ Soucy, Second Wave, S. 84ff.; zum Wandel der SF nach dem Tod von Coty vgl. Müller, Ambition, S. $238 \mathrm{f}$.

38 Demain la France, S. 269.

${ }^{39}$ Maurras, Enquête sur la monarchie, S. 552.

40 Vgl. Soucy, Second Wave, S. 89.
} 
wird nicht Mensch gegen Mensch und Klasse gegen Klasse kämpfen, sondern Volk gegen Regime. ${ }^{" 11}$ Abgesehen von diesen rhetorischen Appellen an den nationalen Konsens fehlten greifbare Forderungen für Arbeiter und Bauern. Die Mischung aus politischem Autoritarismus und wirtschaftlichem Freiraum bot vielmehr attraktive Rahmenbedingungen für Selbständige und mittelständische Unternehmer. Mit diesen Forderungen entpuppten sich die jungen Rechten entgegen ihren Bekundungen als Wirtschaftsliberale der alten Schule, die staatlichen Eingriffen weitgehend mißtrauisch gegenüberstanden und die an die Selbstregulierung des Marktes glaubten ${ }^{42}$.

Mit einem ähnlichen Programm umwarb die Solidarité française nach dem Tod ihres Mäzens François Coty im Juli 1934 auch die dynamischen Berufsgruppen des neuen Mittelstandes, Angestellte, mittelständische Unternehmer und Manager, die sich in wachsendem Maße in den Führungskadern der Bewegung engagierten, während zugleich ihre Anhängerschaft in den kleinbürgerlichen Kreisen zurückging. Unter der Führung von Jean Renaud gewannen auch die Intellektuellen der Jeune Droite an Einfluß in der Bewegung, die nun zunehmend radikale Umsturzpläne propagierte ${ }^{43}$.

Nach den Februarereignissen vervielfachte der Ordre Nouveau innerhalb weniger Wochen seine Aktivitäten und suchte Kontakte zu benachbarten Gruppierungen. Ein neues Forum wurde ins Leben gerufen: der Februar-Club, der nach den Vorstellungen des Ordre Nouveau weiteren Umsturzversuchen zum Erfolg verhelfen sollte: „Damit sie erfolgreich sind, brauchen sie, was den Februartagen gefehlt hat: eine taktische Vorbereitung, ein Ziel, eine Doktrin. ${ }^{44}$ Neben dem Ordre Nouveau versammelten sich dort ehemalige Mitarbeiter von Plans, darunter Le Corbusier, die Nouvelles Equipes von Christian Pineau, einem Gewerkschaftler mit korporatistisch-antiparlamentarischen Interessen, die Action publique von Jacques Arthuys, der zusammen mit Georges Valois den Faisceau gegründet hatte, und der 1933 gegründete Front national-syndicaliste von Pierre Andreu und Jean Le Marchand. Le Marchand, der die Action française 1930 verlassen hatte, unterhielt Kontakte zu den ehemaligen Mitarbeitern der Réaction. Andreu war ein Anhänger des revolutionären Syndikalismus aus der Schule Georges Sorels und näherte sich von dieser Seite der extremen Rechten ${ }^{45}$. In seinen programmatischen Zielen orientierte sich der Club an der Ideologie des Ordre Nouveau. Er stand prinzipiell allen Sympathisanten offen, jedoch unter der Voraussetzung, daß sie dessen personalistische und föderalistische Ziele akzeptierten ${ }^{46}$. Offensichtlich aber waren die Interessen

41 Demain la France, S. 448.

42 Ebenda, S. 176ff., $192 \mathrm{ff}$.

${ }^{43}$ Soucy, Second Wave, S. 62, 87; Müller, Ambition, S. 238f.; vgl. zur Karriere von Maxence innerhalb der SF: APP Ba 1960, Bericht vom 1.7. 1935: Assistent beim 4. Korporatistenkongreß der SF; ebenda, Bericht vom 9.11. 1935: Mitglied des SF-Direktionskomitees.

${ }^{44}$ Club de Février, in: ON 10, April 1934, S. II, derselbe Aufruf auch in: Lutte des Jeunes 5, 25.3.1934, S. 1 .

${ }^{45}$ Andreus Interesse für wirtschaftliche Fragen hatte Georges Valois geweckt. Andreu gehörte zu den Mitarbeitern von Esprit (1934), Lutte des Jeunes (1934), anschließend ging er zum Homme Nouveau (1935) und wechselte 1936 in das Lager der extremen Rechten, schrieb für Combat und Doriots Zeitschrift Emancipation Nationale; Andreu, Rouge, S. 66ff. Vgl. ders., Revoltés, S. 48. Le Marchand gehörte zur AF-Studentenbewegung, war 1930 ausgetreten und hatte das RéactionManifest unterzeichnet; vgl. APP Ba 1895, Bericht vom 1.3. 1930.

${ }^{46}$ NN, Des Jeunes fondent le Club de Février, in: Lutte des Jeunes, H. 6, 1934, S. 1; vgl. Andreu, Rouge, S. 92; ders., Revoltés, S. 47f. 
der Gruppen zu verschieden, um einen dauerhaften Zusammenhalt zu garantieren. Nur mit den von Christian Pineau geleiteten Nouvelles Equipes setzte der Ordre Nouveau die Zusammenarbeit in verschiedenen syndikalistischen Kreisen fort und entwickelte vor allem auf wirtschaftspolitischem Gebiet eine gemeinsame Zielsetzung ${ }^{47}$.

Der Ordre Nouveau gründete darüber hinaus eine eigene Ligue d'action, die über ein eigenes Organ mit dem Titel Nous voulons verfügte. Doch abgesehen von einigen Konferenzen ist von den Aktivitäten dieser Organisation nichts bekanntgeworden. Das Verbandsorgan mußte nach wenigen Nummern sein Erscheinen wieder einstellen, und bereits im Juni des Jahres beklagte Marc die Agonie der Liga. Dennoch hatten diese Versuche, die Basis der Gruppierung zu erweitern, einen gewissen Erfolg. Sie brachten dem Ordre Nouveau einige neue Mitarbeiter und Sympathisanten, die - Robert Aron zufolge - vom kommunistischen Arbeitermilieu bis hinein in die Kreise der Parlamentarier reichten ${ }^{48}$.

Durch diese Aktivitäten veränderte sich die Struktur und personelle Zusammensetzung. Gründungsmitglieder wie Jean Jardin, Daniel-Rops, aber auch Alexandre Marc schrieben seither seltener. In diesen Fällen dürften berufliche und finanzielle Gründe den Ausschlag gegeben haben. Marc und Daniel-Rops arbeiteten seit Anfang 1934 für das von den Dominikanern in Juvisy herausgegebene Wochenblatt Sept, das mit einer durchschnittlichen Auflage von 50-60000 Exemplaren bald zur einflußreichsten katholischen Zeitschrift wurde ${ }^{49}$. Darüber hinaus leitete Daniel-Rops seit 1937 die Reihe Présences im Verlag Plon. Jardin widmete sich verstärkt seiner Karriere bei der französischen Eisenbahngesellschaft ${ }^{50}$, und auch Denis de Rougemont mußte seine Mitarbeit

${ }^{47}$ Pineau schrieb - unter dem Pseudonym Boland - für Ordre Nouveau und später für das von Aron herausgegebene Blatt $A$ nous la Liberté. Auch in den Nouveaux Cabiers arbeiteten die Mitarbeiter beider Bewegungen wieder zusammen. Vgl. Brief Arons an Rougemont, 2.6. [1934]; Brief Marcs an Rougemont, 29.6. [1934] (Pseudonym Boland). Der Brief ist fälschlicherweise nachträglich auf 1936 datiert, Archives Rougemont, Neuchâtel, Ordre Nouveau, Correspondance; vgl. die Ankündigung einer öffentlichen Veranstaltung im Quartier Latin, Salle des Hautes Etudes am 24. 4. 1934, in: Lutte des Jeunes 9, 21. 4. 1934, S. 2.

48 Jacques Dalbon, ein Cousin von Robert Aron, Jacques Lassaigne und Emile Pillias schrieben für Ordre Nouveau, René-Philippe Millet wurde Geschäftsführer des Bulletin ON, vgl. die Namen des Direktionskomitees der Liga in ON 9, März 1934, S. III; Ordre Nouveau, Ou nous en sommes, in: ON, H. 14, 1934, S. III. Auch einige frühere Mitarbeiter der Bewegung wie Gabriel Rey und Jacques Naville tauchten in der Liga wieder auf: Briefe Arons an Rougemont vom 2. 6. [1934] und Marcs an Rougemont vom 29. 6. [1934], Archives Rougemont, Neuchâtel, Ordre Nouveau: Correspondance; vgl. Andreu, Rouge, S. 92. Im Renault-Werk in Puteaux hatte die Bewegung einen Kreis von kommunistischen Arbeitern für ihre Ideen interessieren können; Brief Arons an Rougemont vom 27.4. 1934, Archives Rougemont, Neuchâtel, Ordre Nouveau: Correspondance.

49 Coutrot, Sept, S. 69. Marc war an der Gründung des Blattes mit beteiligt, vgl. Brief Marcs an Rougemont vom 26. 1. [1934], Archives Rougemont, Neuchâtel, Ordre Nouveau: Correspondance. Marc veröffentlichte dort unter dem Pseudonym Scrutator eine politische Wochenchronik und die Presseschau, auch Daniel-Rops verfügte über eine regelmäßig erscheinende Kolumne.

50 Er war Privatsekretär des Direktors Raoul Dautry; Brief Marcs an Rougemont, 29. 6. [1934], Archives Rougemont, Neuchâtel, Ordre Nouveau, Correspondance; vgl. Assouline, Eminence grise, S. 31; Badoui, Dautry, S. $175 f$. 
während seines Lektorats in Frankfurt 1935-1936 einschränken. An ihre Stelle traten zahlreiche neue, meist jüngere Mitarbeiter. Xavier de Lignac, ein Absolvent von Sciences Politiques, leitete zusammen mit Mireille Dandieu, der Schwester Arnauds, das Sekretariat der Zeitschrift ${ }^{51}$. Mit dem Zustrom neuer Mitarbeiter wie Pierre Prévost ${ }^{52}$, Albert und Louis Ollivier ${ }^{53}$ veränderten sich auch die inhaltlichen Schwerpunkte. Unter ihrem Einfluß rückte die Gruppierung von den stark ideologisch orientierten Debatten ab und orientierte sich eher an tagespolitischen Themen. Insbesondere die Wirtschaftspolitik geriet seitdem mehr ins Blickfeld. Die neuen Anhänger sympathisierten mit syndikalistischen Ideen, lehnten jedoch den reformistischen Kurs der C.G.T. entschieden ab ${ }^{54}$.

Ihre Bereitschaft zur Beteiligung an einem neuerlichen Umsturz, soweit er mit einem konstruktiven Erneuerungsprogramm verbunden war, hatten die Mitarbeiter des Ordre Nouveau unmittelbar nach dem Februaraufstand bekundet. Kurze Zeit später schien sich tatsächlich eine Zusammenarbeit mit den Feuerkreuzlern anzubahnen. Zwei Mitarbeiter des Ordre Nouveau, Robert Loustau und Robert Gibrat, waren nach den Februarereignissen in die Bewegung des Oberst François de la Rocque eingetreten ${ }^{55}$. Auch die Stellungnahmen weiterer Mitarbeiter zeugten von einer kritischen Sympathie für die Feuerkreuzler. René Dupuis nahm die Bewegung von seiner Kritik an den übrigen Ligen ausdrücklich aus: Während Francisme, Solidarité française und Bergerys Front commun den Kampf der Parteien um Anhänger nur mit anderen, gewalttätigen Mitteln fortsetzten, versprächen die Feuerkreuzler ein konstruktives Sammlungsprogramm, das das Gemeinwohl im Blick habe. Dupuis würdigte diese Absicht, doch kritisierte er das Fehlen einer schlüssigen Doktrin. Er verdeutlichte die Widersprüche und dogmatische Schwäche von La Rocques Werk Service Public und betonte, daß eine Sammlungsbewegung nur mit dem Programm des Personalismus erfolgreich sein könne ${ }^{56}$. Robert Aron schätzte an der Bewegung besonders ihre Feindschaft zum Parlamentarismus und sah in ihr sogar "den Kern und die Konzeption des zukünftigen Frankreich“"57.

Über den faschistischen Charakter der Feuerkreuzler gibt es kontroverse Ansichten. Die französische Zeitgeschichtsforschung schließt sich der These René Rémonds an, nach der die Bewegung zu stark in der Tradition der extremen Rechten verwurzelt war,

\footnotetext{
51 Archives Rougemont, Neuchâtel: ON: pièces diverses. Protokoll einer Organisationssitzung bei Aron vom 20.4. 1936; vgl. Ganne, Qu'as-tu fait de ta jeunesse?, S. 8.

52 Er war Angestellter in einer Fabrik in Straßburg; vgl. Prévost, Lettre, in: Lutte des Jeunes, H. 16, 1934, S. 3; zusammen mit Chevalley und Lignac pflegte er Kontakt mit Georges Bataille und besuchte regelmäßig die Veranstaltungen des Collège de sociologie; Prévost, Rencontres, S. 11-35.

${ }^{53}$ Sie waren möglicherweise über die Vermittlung von Daniel Halévy, in dessen Salon sich regelmäßig Aron, Rougemont und Marc trafen, zu Ordre Nouveau gestoßen; vgl. Aron, De Gaulle, S. 42; Rougemont, Journal d'une Epoque, S. 365.

${ }^{54}$ Lignac, Syndicalisme, Socialisme et Révolution, in: ON, H. 34, 1936, S. 23-29; A. Ollivier, L'émancipation des travailleurs, in: ON, H. 41, 1937, S. 18-23.

55 Aron hat den Kontakt der beiden zu ON vermittelt: Aron, Fragments, S. 105; Pucheu, Ma vie, S. 72; Andreu, Revoltés, S. 41.

${ }_{56}$ De Cultures [Dupuis], Bilan de Février, in: ON, H. 18, 1935, S. $1 \mathrm{ff}$.

57 Aron, Dictature de la Liberté, S. 228. Das Werk wurde zwischen April 1934 und Juli 1935 verfaßt.
} 
um die den faschistischen Bewegungen eigene Dynamik zu entwickeln ${ }^{58}$. Mit Recht betonen hingegen William Irvine und Robert Soucy, daß die Gräben zwischen der traditionellen Rechten und faschistischen Bewegungen nicht so tief waren, wie dies die Anhänger der Rémond-These behaupten. Vielmehr sehen sie gerade im Bonapartismus den Nährboden für einen spezifisch französischen Faschismus. Ein Beleg für diese Ansicht ist die erhebliche Fluktuation zwischen den Feuerkreuzlern und Anhängern anderer, auch von Rémond als faschistisch eingestufter Ligen wie dem Francisme und der Solidarité française. Soucy sieht im Kult des Militarismus und dem militanten Antikommunismus der Feuerkreuzler durchaus radikale und dynamische Züge, die über die traditionelle Rechte hinausweisen ${ }^{59}$. Als Anführer des Februaraufstandes waren sie die Hauptfeinde der „antifaschistischen“ Propaganda der Volksfront-Bewegung. Bei der Linken waren sie vor allem deshalb gefürchtet, weil sie in kürzester Zeit eine große Anzahl von Anhängern mobilisieren konnten und bei den Aufmärschen gerne ihre paramilitärische Macht zur Schau stellten. Nicht zuletzt ihr großer Zulauf nährte die Furcht vor einem faschistischen Putsch. Innerhalb von eineinhalb Jahren nach den Februarereignissen hatte die Liga ihre Anhängerzahl versechsfacht. Im Juli 1935 waren es rund $240000^{60}$.

Auch Oberst de La Rocque war an einer Zusammenarbeit mit dem Ordre Nouveau interessiert. Einige Zeit hatte er erwogen, die Gruppierung zum geistigen Führungsstab seiner Feuerkreuzler zu machen. Doch bei einer persönlichen Unterredung traten die Diskrepanzen deutlich zutage: Beide Seiten beschuldigten sich gegenseitig der Arroganz und Unnachgiebigkeit. Als La Rocque seine Anhängerzahlen in die Waagschale warf, konterte Aron: „Die Gasversorgung hat mehr Abonnenten.“61 Doch auch nach dieser gescheiterten Verständigung blieb der Ordre Nouveau den Feuerkreuzlern gewogen. Rougemont verteidigte sie gegen den Vorwurf des Faschismus und warf der Volksfront vor, ihre ganze Sammlungspolitik auf einem konstruierten Feindbild aufzubauen. Rougemont sah in dem „braven Oberst“ vielmehr einen Vertreter jener Konservativen, deren Wertvorstellungen sich von denjenigen der Faschisten grundlegend unterschieden: „Wertvorstellungen von ,Familienvätern', wiederbelebte bürgerliche Moral, in Wahrheit sind wir weit entfernt von jeder Art von sogenanntem verborgenen oder offen bekunde-

${ }^{58}$ Das Verhalten La Rocques am 6. Februar hatte Rémond zufolge deutlich gemacht, daß der Anführer der Liga vor der Übernahme der Macht im entscheidenden Augenblick zurückschreckte. Auch Nolte spricht ihr das "faschistische Minimum“ ab und bescheinigt ihr mangelnde Radikalität in Ideologie und Praxis. La Rocque distanzierte sich von der aggressiven politischen Praxis eines Mussolini und Hitler. Die Aufmärsche der Feuerkreuzler hatten eher das Ziel, die kommunistischen Revolutionäre einzuschüchtern, als selbst die Macht zu ergreifen. La Rocques Vorstellungen kamen einem autoritären Staatsideal näher als einem faschistischen; Rémond, Droites, S. 206ff.; Machefer, Ligues, S. 32; Milza, Fascisme français, S. 133; Nolte, Die faschistischen Bewegungen, S. 294.

${ }^{59}$ Auch die eher moderate Feindschaft zum Parlamentarismus war nach Ansicht Soucys eher von taktischen Erwägungen bestimmt. Im Unterschied zu den meisten übrigen Ligen waren jedoch antisemitische Ressentiments bei den Feuerkreuzlern kaum zu vernehmen. Zumindest in den dreißiger Jahren verurteilte $L a$ Rocque den nationalsozialistischen Rassismus ausdrücklich und nahm Juden in die Reihen seiner Bewegung auf; Soucy, French Fascism and the Croix de Feu, S. 165ff.; Irvine, Fascism in France, S. 278f.

${ }^{60}$ Allein in der Pariser Region hatten sie 30- bis 35000 Anhänger, APP Ba 1901, Bericht vom 10.7. 1935; Soucy nennt 228000 im August 1935: Croix de Feu, S. 159.

${ }^{61}$ Aron, Fragments, S. 108; Gespräch mit Alexandre Marc am 4.12. 1994. 
ten Faschismus." ${ }^{62}$ Aber dieses Festhalten an den Werten der bürgerlichen Rechten verhinderte vorläufig die Zusammenarbeit mit dem Ordre Nouveau. Ein neuer Annäherungsversuch war damit nicht ausgeschlossen, aber an die Bedingung geknüpft, daß die Feuerkreuzler ihren paternalistischen Kosmos verließen und sich linken Sozialreformen öffneten.

Mit den Beiträgen zur Planwirtschaft hat sich der Ordre Nouveau an einer Debatte beteiligt, die vor allem auf der Linken in den Jahren 1934 und 1935 lebhaftes Interesse fand. Ausgelöst wurde sie durch den Plan der Arbeit des belgischen Sozialisten Hendrik de Man, dessen Überlegungen im November 1933 in Frankreich bekannt wurden und der wirtschaftspolitischen Diskussion maßgebliche neue Impulse gaben. Die Unruhen des Februar hatten diesen Überlegungen weitere Aktualität verliehen, da die Protagonisten die Planwirtschaft als Mittel der politischen Stabilisierung betrachteten. In der Folge entwickelten zahlreiche Gruppierungen der Linken, darunter die Neosozialisten, der Front social, die C.G.T. und die Gruppe des 9. Juli, ein planwirtschaftliches System. In Anlehnung an den „Plan de Man“, wie der „Plan van de Arbed“ in Frankreich auch genannt wurde, forderten die französischen „Planisten“ eine gemischte Wirtschaft, die noch einen Rest von freiem Kräftespiel erlaubte, in der zugleich aber die Autorität des Staates über die Wirtschaft gesichert war. Darin unterschied sich dieses System grundsätzlich von dem sowjetischen, das die Marktwirtschaft vollkommen unterband. Der „Plan de Man" lieferte erstmals das theoretische Fundament für eine Strukturreform des liberalen Kapitalismus, die nicht in einen staatlich dirigierten Sozialismus mündete ${ }^{63}$. Die Debatte um den Plan setzte bei der sozialistischen Linken zugleich vollkommen neue Akzente: Nicht mehr der Kampf, sondern die Versöhnung der Klassen bildete das oberste Ziel der Politik. Die Anhänger des Plans waren somit zu einer prinzipiellen Revision sozialistischer Maximen bereit. Höchste Priorität hatte nicht mehr die Klasse, sondern die Nation.

Unter dem Einfluß Robert Gibrats und Robert Loustaus, zwei Anhängern der Feuerkreuzler, nahm der Entwurf des Ordre Nouveau konkrete Formen an. Die beiden Polytechniker hatten ihre wirtschaftspolitischen Kenntnisse in der Auseinandersetzung mit den neoliberalen und sozialistischen Reformern des Studienkreises X-crise vertieft. Nach dem Vorbild des belgischen Plans ging auch derjenige des Ordre Nouveau von zwei Wirtschaftssektoren aus: der Kontrolle des Plans sollten alle Bereiche in Industrie und Landwirtschaft unterworfen sein, die für die Grundversorgung mit Kleidung, Möbeln, Nahrung und Wohnungsbau zuständig waren. Für alle anderen Güter galten die Gesetze der freien Marktwirtschaft ${ }^{64}$.

Damit aber waren die Gemeinsamkeiten bereits erschöpft. Anders als die übrigen Pläne sollte derjenige des Ordre Nouveau an die Ablösung der parlamentarischen Herrschaft gekoppelt sein. Die Gruppierung sah in dem Plan kein vorübergehendes Mittel der Krisenbewältigung, sondern vielmehr ein dauerhaftes Instrument der Wirtschaftskontrolle. Den Charakter des belgischen Plans kritisierte die Gruppierung: für sie war die Planwirtschaft kein bloßes Korrekturinstrument, sondern die Voraussetzung für

\footnotetext{
${ }^{62}$ Rougemont, Où en est la France? in: ON, H. 24, 1935, S. 1ff., Zitate S. 1 und S. 3.

${ }^{63}$ Dodge, Beyond Marxism, S. 136, Nachdruck des Plans der Arbeit S. 232-236.

${ }^{64} \mathrm{Vgl}$. Brun, Technocrates, S. 33ff.; Gibrat/Loustau, Economie libre et économie planée, in: ON, H. 10, 1934, S.6f.; Hélisse, Le plan de Man, in: ON, H. 22/23, 1935, S. 37-42.
} 
eine dauerhafte Abkehr von Kapitalismus und parlamentarischer Demokratie. Charakteristisch für diese Sichtweise ist ein weiteres Element, das in den meisten anderen planwirtschaftlichen Konzepten fehlt oder zurücktritt: Die Produktion in der kontrollierten Zone sollte nicht nur die Grundbedürfnisse decken, sondern die Grundlage des wirtschaftlichen Wachstums bilden ${ }^{65}$. Somit war die Planwirtschaft beim Ordre Nouveau auch weit mehr als nur ein Instrument der Kontrolle, sie diente vielmehr der Effizienzsteigerung der Volkswirtschaft.

Diese Form der gemischten Planwirtschaft und die antikapitalistische Programmatik zielten - ähnlich wie bei der Jeune Droite - nicht auf Enteignung und Verstaatlichung, sondern nur auf die Zerschlagung der „parasitärer Organismen“: der Aktiengesellschaften, Großbanken und Börsen ${ }^{66}$. Privateigentum hingegen sahen die Mitarbeiter des Ordre Nouveau als Voraussetzung einer menschenwürdigen Existenz an. Ausdrücklich betonte der Ordre Nouveau daher das Leistungsprinzip, das dem Arbeitnehmer eine Beteiligung an den Früchten seiner Arbeit versprach und ihn auf diese Weise in die unternehmerische Verantwortung einband. Der Status des Lohnempfängers sollte zugunsten einer flexiblen, am Gewinn des Unternehmens orientierten Bezahlung aufgegeben werden. Die nötigen Leistungsanreize schuf die Beteiligung des Arbeitnehmers am Produktivvermögen. Neben der größeren Eigenverantwortung hatte dieses Modell auch die stärkere Bindung des Arbeitnehmers an die Zukunft des Unternehmens zur Folge ${ }^{67}$. Ein weiterer Neuansatz, der auf die Ideen des Bergbauingenieurs Loustau zurückging, war die Umgestaltung der Unternehmensführung, die man durch eine Art Unternehmensvorstand ersetzen wollte, in dem die Arbeiter und Techniker gemeinschaftlich mit dem Unternehmer die Führung des Betriebes übernehmen und auch eine Mitspracherecht bei der Schlichtung von Arbeitskonflikten haben sollten ${ }^{68}$.

Innerhalb der Planwirtschaft erhielt auch der Arbeitsdienst einen neuen Stellenwert. Ursprünglich gedacht als flexibles Instrument zur gleichmäßigen Verteilung der Lasten der "nichtqualifizierten“ Arbeit, gewann er nun entscheidende Bedeutung für die Erfüllung des Plans. Neben dem Gedanken der sozialen Gerechtigkeit enthielt er eine klare wirtschaftliche Zielsetzung: Jeder Bürger sollte seinen Beitrag von maximal 18 Monaten leisten, um ein Anrecht auf sein Existenzminimum zu erwerben und darüber hinaus die Volkswirtschaft zu stärken ${ }^{69}$. In diesem Fall sind die Mitarbeiter des Ordre Nouveau nicht bei der Planung geblieben, sondern haben selbst ein Beispiel dafür gegeben, wie der Arbeitsdienst verwirklicht werden konnte: Im Sommer 1935 haben sie Fließbandarbeiter in vier Fabriken in Beauvais und Paris für zwei Wochen von ihrer Tätigkeit abgelöst, damit diese in den Genuß eines bezahlten Urlaubs kamen, der ihnen bis dahin ebensowenig zustand wie eine auf vierzig Wochenstunden beschränkte Arbeitszeit. Dieses Experiment war gedacht als "praktischer Beweis, daß eine wirkliche Solidarität zwischen

${ }^{65}$ Marc, Conditions de tout plan, in: ON, H. 22/23, 1935, S. 6.

${ }_{66}$ Nous voulons, in: ON, H. 9, 1934, S. 16.

67 Ebenda, S. $21 \mathrm{ff}$.

${ }^{68}$ Loustau, Un exemple concret: la mine, in: ON, H. 7, 1934, S. 25-32; Dupuis/Marc, Corporation, in: ON, H. 10, 1934, S. $17 f$.

${ }^{69}$ Gibrat, L'organisation du Service Civil, in: ON, H. 7, 1934, S. 17-24; Nous voulons, in: ON, H. 9, 1934, S. 18; A. und L. Ollivier, La valeur morale du travail, in: ON, H. 20, 1935, S. 8-12; vgl. Aron/Dandieu, Révolution nécessaire, S. 251. 
den Klassen in Zukunft an die Stelle des Klassenkampfes treten kann"70. Deutlich trat hier der zentrale Gedanke der Solidargemeinschaft hervor, der die gesamte planwirtschaftliche Diskussion bestimmte. Ziel des Arbeitsdienstes sollte es künftig sein, alle Grundbedürfnisse wie Essen, Kleidung, Wohnen auf diese Weise abzudecken.

Es fanden sich genug Freiwillige, um insgesamt vierzig Arbeitern einen bezahlten Urlaub zu ermöglichen. Dieses Experiment wurde von seiten der Arbeiter ebenso positiv aufgenommen wie von den Arbeitgebern ${ }^{71}$. Das Eintreten für die Planwirtschaft ließ sich mit der angestrebten föderalistischen Ordnung, die auf spontanen kommunalen $\mathrm{Zu}$ sammenschlüssen beruhen sollte, nur schwer vereinbaren ${ }^{72}$. Auch wenn der Wille aufrichtig war, die Macht des Staats soweit wie möglich einzuschränken, so trug doch die Planwirtschaft unweigerlich dazu bei, dessen Macht zu stärken. Wie läßt sich die Forderung nach einem „schlanken“ Staat mit seiner Planungskompetenz in allen zentralen Bereichen der Wirtschaft vereinbaren? Der Gedanke einer Revolution von unten mit weitgehender Selbstverwaltung der Kommunen und Regionen stand in deutlichem Widerspruch zu den planwirtschaftlichen Zielen, die den nationalen Interessen den Vorrang gaben. Dies um so mehr, als für die Zusammensetzung des Wirtschaftsrates ebenso wie für die des ihm gleichgestellten Verwaltungsrates weitgehend das Prinzip der Kooptation, also der Selbstrekrutierung, galt, so daß er keiner effektiven Kontrolle von unten unterlag. Über dem Wirtschaftsrat stand nur der höchste Rat (conseil suprême), der seine Mitglieder völlig frei ernennen konnte. Seine Aufgabe war es, alle Organe der Föderation zu kontrollieren und Streitigkeiten zwischen ihnen zu schlichten. Als oberster Wächter besaß er eine normative Autorität, die es ihm erlaubte, die Staatsziele zu bestimmen $^{73}$. Darüber hinaus besaß der höchste Rat die „direkte und souveräne Entscheidungsbefugnis in allen Konflikten über Grundprinzipien und lebenswichtige Interessen der Föderation “74. Er war also eine Art oberstes Verfassungsgericht, ausgestattet mit den höchsten Befugnissen der Exekutive. Demokratische Verfahren waren hingegen nur an der Basis zugelassen: Die kommunalen Räte wurden zum Teil direkt von allen Bewohnern einer Kommune gewählt (andererseits wiederum durch Kooptation bestimmt). Nur als unmittelbare und direkte Form hatte der Begriff der Demokratie einen Sinn - als Beispiel nannte Rougemont an anderer Stelle die kleinen Landgemeinden seiner Schweizer Heimat ${ }^{75}$.

Es bleibt zumindest fraglich, wie weit eine solche Verfassung in der Praxis föderalistisch sein konnte, die von unten fast keine Kontrollmöglichkeiten bot und in der eine quasi-absolutistische Instanz wie der höchste Rat das verbindliche Interpretationsmonopol für alle Normen- und Instanzenkonflikte besaß. Wer kontrolliert schließlich die höchste Instanz, wo es keine Gewaltenteilung und keine demokratische Legitimation

70 Ordre Nouveau, La relève du travail, in: ON, H. 20, 1935, S. 2.

71 Aron, Notre expérience de service civil, in: Bulletin ON, Nr. 5, 15. 10. 1935, S. 4.

72 Chevalley/Glady [Marc], La mort des Partis, in: ON, H. 4, 1933, S. 19-27, S. 24.

${ }^{73}$ Marc, L'état sans majuscule, in: ON, H. 14, 1934, S. 32; Rougemont, Qu'est-ce que l'autorité?, in: ON, H. 31, 1936, S. 1-5.

${ }^{74}$ Eine Skizze der politischen und wirtschaftlichen Organisation findet sich in ON, H. 9, 1934, S. Iff. Zitat S. VII, wieder abgedruckt in Aron, Dictature, S. $264 \mathrm{ff}$.

${ }^{75}$ Rougemont, Plébiscite et démocratie, in: ON, H. 30, 1936, S. 21-25, hier S. 23. Als Größenordnung nannte er „einige Tausend“ Bürger. 
mehr gibt? Rougemont gab darauf eine eindeutige Antwort: Diese Elite werde nur von ihrem eigenen Gewissen kontrolliert. In seinem 1934 erschienenen Werk Politique de la Personne legitimierte Rougemont den Herrschaftsanspruch einer neuen, von personalistischen Ideen durchdrungenen Führungsgruppe. Ihren Anspruch auf Autorität bezog sie aus einer persönlichen Berufung durch Gott, die Rougemont aus der kalvinistischen Lehre ableitete. Diese Elite hatte damit das Recht, ihre Ziele auch gegen den Willen der Mehrheit durchzusetzen: „[...] das lebendige Zentrum eines Landes liegt nicht in einer Organisation, die Zwang ausübt. Es sollte vielmehr in jedem mündigen Bürger selbst liegen, auch wenn es sich - wie dies der Fall ist - um eine Minderheit handelte. " ${ }^{\text {"76 }}$

\section{Faschistischer Korporatismus}

Ein Wirtschaftswissenschaftler bezeichnete 1934 etwas vorschnell das zwanzigste Jahrhundert als "Jahrhundert des Korporatismus", charakterisierte damit aber zutreffend den Stellenwert der Debatte in der Zwischenkriegszeit ${ }^{77}$. Bevor der Korporatismus durch seine autoritären Varianten diskreditiert wurde, besaßen die Ideen nicht nur bei zahlreichen konservativen, sondern auch bei sozialreformerischen Wirtschafts- und Gesellschaftstheoretikern große Anziehungskraft. Die Vereinnahmung genossenschaftlicher Ideen durch die diktatorischen Systeme hat die Sicht auf die weniger autoritären Varianten des Korporatismus lange Zeit verstellt. Erst mit der Diskussion um den „Neokorporatismus" Mitte der siebziger Jahre konnte der Begriff von seinem pejorativen Beiklang teilweise befreit werden und der Blick für die Vielfalt der Ansätze frei werden, die aus der Einsicht in die begrenzte Fähigkeit der Selbstregulierung des Kapitalismus entstanden $\operatorname{sind}^{78}$.

Zweifellos hatte Italien mit der Einführung des Korporativsystems in der Carta del lavoro 1927 eine Pionierrolle übernommen. Am italienischen Modell orientierten sich insbesondere diejenigen Länder, für die der Faschismus auch als politisches System zum Vorbild wurde, so Portugal unter Salazar und Österreich unter Dollfuß. Aber Papst Pius XI. hatte in der Sozialenzyklika Quadragesimo Anno (1931) das Prinzip der berufsständischen Organisation auch für die Katholiken als verbindliche Sozialordnung verkündet. So konnte es nicht verwundern, daß nach den Lateranverträgen zumindest nach außen hin der Eindruck der Verständigung zwischen Staat und Kirche über die Grundprinzipien der Gesellschafts- und Wirtschaftspolitik entstehen konnte ${ }^{79}$.

Gerade die inneren Reformen des italienischen Nachbarn beobachtete die nonkonformistische Generation mit großer Aufmerksamkeit, bevor der Abessinienkrieg die impe-

\footnotetext{
${ }^{76}$ Rougemont, Politique, S. 22 (Zitat), 64; zum Begriff der vocation bei Rougemont vgl. Ackermann, A la recherche, S. $427 \mathrm{ff}$.

77 Manoilesco, Le siècle du corporatisme.

78 Werner Abelshauser diagnostiziert für die deutsche Entwicklung im Industriezeitalter das Nebeneinander von autoritär ständestaatlichen und postliberalen Richtungen im deutschen Korporatismus; Abelshauser, Freiheitlicher Korporatismus, S. 148f. und ders., The First Post-Liberal Nation, S. 285-318; noch ganz im pejorativen Sinn gebraucht Mayer-Tasch den Begriff: MayerTasch, Korporativismus und Autoritarismus.

79 Zur Sozialenzyklika vgl. Monzel, Katholische Kirche, S. 268-282.
} 
rialistische Seite der Diktatur offenbarte. Nicht nur die Jeune Droite verglich ihren von der katholischen Soziallehre inspirierten Korporatismus mit der Verwirklichung des Systems jenseits der Alpen, auch für den Ordre Nouveau besaß der italienische Weg aus dem Kapitalismus intellektuelle Anziehungskraft. Zwar war die Haltung der Gruppierung von einer kritischen Distanz bestimmt, doch gab es ein deutliches Interesse an einem Dialog. Robert Aron gehörte neben den Mitarbeitern der neosozialistischen Zeitschrift Homme Nouveau, Pierre Andreu, Paul Marion und Georges Roditi, zu den Organisatoren einer Informationsveranstaltung über den faschistischen Korporatismus im Mai 1935. Mit ihnen kam eine ganze Reihe von Vertretern der nonkonformistischen Intellektuellenzirkel nach Rom: Mounier, Ulmann, Galey für Esprit, Dupuis und Chevalley für den Ordre Nouveau, Georges Viance als Vertreter des katholischen Korporatismus, Achille Bouxin für die Landfront (Front paysan), Pierre Ganivet, der Herausgeber der syndikalistischen Zeitschrift Homme réel, Pierre Gimon für die Jeunesses Patriotes und Fabrègues für die Jeune Droite.

Vier Tage lang hatten sie dort als Gäste des faschistischen Kulturinstituts die Gelegenheit, mit namhaften Vertretern aus Politik, Wirtschaft und Wissenschaft zu diskutieren. Zur italienischen Delegation gehörten Giuseppe Bottai, einer der wichtigsten innenpolitischen Berater Mussolinis und Theoretiker des Korporativsystems, Razza, der Minister der Korporationen, Landwirtschaftsminister Rossoni, der Philosoph Ugo Spirito sowie Robert Michels und Hubert Lagardelle ${ }^{80}$.

Ganz offensichtlich bestätigte dieses Treffen bereits vorher bestehende Abneigungen und Sympathien - aber die direkte Auseinandersetzung mit den Vertretern des faschistischen Korporatismus verstärkte die unterschiedlichen Ansichten innerhalb der französischen Delegation. Zum Schluß standen sich Bewunderer und Gegner des faschistischen Korporatismus in scharfen Frontlinien gegenüber. Zur ersten Fraktion gehörten die Jeune Droite, vertreten durch Fabrègues, und die Neosozialisten des Homme Nouveau (Andreu, Roditi, Marion). Paul Marion sah die einzige Lösung der gegenwärtigen Krise in einem starken Staat, der seiner Ansicht nach nur durch die Einführung des Korporativsystems verwirklicht werden konnte. Er verwarf entschieden die Idee des Klassenkampfes. Das italienische Experiment der Versöhnung der Klassen war Marion zufolge geglückt, doch betonte er zugleich, daß Frankreich aufgrund anderer nationaler Gegebenheiten seinen eigenen Weg finden müsse ${ }^{81}$. Auch Pierre Andreu vertiefte in der Folge seine Kontakte nach Italien und wurde ein ausgesprochener Bewunderer des faschistischen Systems ${ }^{82}$.

Fabrègues gab zwar keine Stellungnahme zu dem Kongreß ab, doch bekundete er weiterhin grundsätzliche Sympathie für den Korporatismus. Seine Argumentation bewegte sich auf der Linie, wie er sie zuvor in der Revue du siècle entwickelt hatte: Autorität und Hierarchie bildeten die gemeinsamen Grundpfeiler der Ideen La Tour du Pins und des italienischen Systems. Bei letzterem mißbilligte er zwar die Omnipräsenz des Staates,

${ }^{80} \mathrm{Vgl}$. zu den Teilnehmern Mounier, Entretiens, Euvres, Bd. 4, S. 570; ders., Esprit au congrès franco-italien sur la corporation, in: Esprit, H. 33, 1935, S. 474-480; Ganivet, Un congrès d'études corporatives à Rome, in: Homme réel, H. 19, 1935, S. 22; Millet, Jeunes Français et jeunes Italiens I, in: Le Temps 11.6. 1935, S. 6; Andreu, Revoltés, S. 48, 65.

${ }^{81}$ Millet, Jeunes Français et jeunes Italiens II, in: Le Temps 20.6. 1935, S. 8.

${ }^{82}$ Andreu, Rouge, S. $96 \mathrm{ff}$. 
doch relativierte er sie durch den Hinweis auf den gemeinsamen Feind, den liberalen $\mathrm{Ka}$ pitalismus ${ }^{83}$.

Der Ordre Nouveau distanzierte sich nach dem Kongreß in aller Schärfe von diesen Sympathisanten des italienischen Systems, denen man „fehlende Einsicht und unzulänglichen Charakter" vorwarf 84 . Unter dem Eindruck der Debatten in Rom wurde die Gruppierung - ähnlich wie die Vertreter des Homme réel und von Esprit - zur erklärten Gegnerin des faschistischen Korporatismus und letztlich des Korporativsystems überhaupt. Die Auseinandersetzung mit dem faschistischen System fiel zwar sachlicher als der Angriff gegen die französischen Kollegen aus, doch auch hier kam der Ordre Nouveau zu deutlichen Schlußfolgerungen: Mussolini habe es nicht vermocht, den Kapitalismus zu überwinden. Nach Ansicht Arons bestand zwischen den Korporationen und einem nach kapitalistischen Maßstäben geführten Großunternehmen nur ein gradueller Unterschied. Dem Faschismus war es Aron zufolge nicht gelungen, die enge Verflechtung von Staat und Wirtschaft zu lösen ${ }^{85}$. Gegenüber Mussolinis Berater Bottai gab er deutlich zu verstehen, daß der staatliche Einfluß einen freiheitlichen Korporatismus verhindere. Aron kritisierte die alles beherrschende Stellung des faschistischen Großrats, dessen Einfluß auf die Kammer der Korporationen und die Gewerkschaften nahezu unbeschränkt war. In die gleiche Richtung ging die Kritik von Esprit, wobei die Zeitschrift sich deutlicher um Verständnis für das italienische Projekt bemühte. Die Vertreter des Homme réel, die an dem Plan der französischen Gewerkschaft C.G.T. mitarbeiteten, ließen sich hingegen wenig von den römischen Absichtsbekundungen beeindrucken. Ganivet bezweifelte nicht so sehr die guten Absichten der italienischen Delegation als vielmehr ihren tatsächlichen Einfluß in einem System, das auf Zensur und Unterdrückung angewiesen war $^{86}$.

\section{Die Sammlungsbewegung der Dissidenten}

Seit dem Sommer 1935 nahmen die Pläne zu einer größeren Sammlungsbewegung verschiedener Organisationen der Linken und der Rechten konkrete Form an. Auch der Ordre Nouveau und Esprit beteiligten sich daran. Gegenüber Chevalley betonte Marc

${ }^{83}$ Fabrègues, Indépendance, in: Combat, H. 2, 1936, S. 21; ders., Où sont vos principes?, in: Combat, H. 4, 1936, S. 53f.

${ }^{84}$ Ordre Nouveau, De la trahison, in: ON, H. 21, 1935, S. III; vgl. auch die Kritik Chevalleys, Un congrès à Rome, in: Bulletin ON, Nr. 3, 15.6. 1935, S. 4.

${ }^{85}$ Aron, Aspects du fascisme, in: ON, H. 18, 1935, S. 21-25. Diese Äußerungen waren das Ergebnis einer Vorbesprechung mit der italienischen Delegation. Vgl. auch Scrutator [Marc], Les sept jours, in: Sept, H. 50, 1935, S. 2. Der Ordre Nouveau gebrauchte zwar auch den Begriff der Korporation, doch in einem Sinn, der mit den Korporatismus-Modellen nichts zu tun hat und sich vielmehr mit dem Begriff des Unternehmens deckte, vgl. Dupuis/Marc, Corporation, in: ON, H. 10, 1934, S. 8-28, hier S. 10f.; "Nous voulons“, in: ON, H. 9, 1934, S. 23.

${ }^{86} \mathrm{NN}$, Esprit au congrès franco-italien sur la corporation, in: Esprit, H. 33, 1935, S. 474-480; Aron, Fausses sorties, in: ON, H. 21, 1935, S. 20f.; Ganivet, Un congrès d'études corporatives à Rome, in: Homme réel, H. 19, 1935, S. 22; Millet, Jeunes Français et jeunes Italiens II, in: Le Temps 20.6. 1935, S. 8. Das Gespräch Arons mit Bottai wird bei Ganivet erwähnt. 
die Notwendigkeit, den strikten „Weder rechts noch links“-Kurs aufrechtzuerhalten, um zu einem Forum für die Dissidenten aller politischen Lager werden zu können ${ }^{87}$.

Insbesondere die planwirtschaftliche Debatte ermöglichte es, die unterschiedlichen Standpunkte zu überwinden und das gemeinsame nationale Interesse in den Vordergrund zu stellen. Zunächst war die Initiative von den Dissidenten der linken Parteien ausgegangen: dem Front social unter der Führung von Bergery, den Neosozialisten unter Déat und den ehemaligen Kommunisten unter der Leitung des Bürgermeisters von Saint-Denis, Jacques Doriot ${ }^{88}$. Sie erhielten im Juli des Jahres Zulauf von rechts durch die Nationalvolontäre (Volontaires Nationaux), die mit La Rocque gebrochen und sich von den Feuerkreuzlern getrennt hatten ${ }^{89}$. Die Spaltung innerhalb der Feuerkreuzler war die Folge eines Richtungsstreits zwischen dem Oberst und einem Führer der Nationalvolontäre, Bertrand de Maud'hui. Letzterer hatte Ende Juni 1935 Kontakt zu Bergery aufgenommen und sich kurze Zeit später von la Rocque losgesagt. Mit ihm verließ ein großer Teil der Führung der Nationalvolontäre die Bewegung. Die Mehrheit dieser Dissidenten war nach dem 6. Februar den Feuerkreuzlern beigetreten und hatte keine ausgesprochen rechtsextreme Vergangenheit ${ }^{90}$. Darunter waren auch die Anhänger des Ordre Nouveau, Loustau und Gibrat, die inzwischen eine Zusammenarbeit mit Esprit anvisierten $^{91}$. Das Projekt wurde von verschiedenen Seiten propagandistisch unterstützt. Der neosozialistische Kreis um den Homme nouveau beteiligte sich an den Verhandlungen. Mit der Parole „Sammlung oder Bürgerkrieg“ setzte sich auch Georges Izard, ein Mitgründer von Esprit und Chef der Troisième Force, für die Sammlung ein. Seit Juli verfügte die Sammlungsbewegung über ein eigenes Wochenblatt Révolution, in dem sich zahlreiche Intellektuelle der extremen Linken und Rechten für die Idee warben ${ }^{92}$.

Seit dem Herbst 1935 schließlich beteiligte sich der Ordre Nouveau zusammen mit Esprit an den Plänen. Im Oktober hatten sich die beiden Gruppen zu einer Aussprache getroffen, anschließend bekundete Mounier in der Novemberausgabe von Esprit seine Bereitschaft zur konstruktiven Mitarbeit. Mounier, der dem Ordre Nouveau ein Abdriften nach rechts vorgeworfen hatte, bemühte sich, die Differenzen auf Unterschiede des Temperaments zu reduzieren, und eröffnete in seiner Zeitschrift die Diskussion über eine „dritte Kraft“, die die Leitlinien einer gemeinsamen Politik erarbeiten sollte. Er plädierte für eine Sammlung auf der Grundlage des „Plans des 9. Juli“, den eine Gruppe junger Intellektueller von den Jeunesses Patriotes bis zu den Neosozialisten unter der Leitung von Jules Romains entworfen hatte. Die weiteren Beiträge dieser Debatte stammten von Loustau, der seine Gründe für den Bruch mit La Rocque erläuterte, und

\footnotetext{
87 Brief Marcs an Chevalley vom Juli 1935, zitiert bei Lipiansky, Ordre Nouveau, S. 85.

88 Burrin, Dérive, S. 160ff.; Soucy, Second Wave, S. $209 \mathrm{ff}$.

${ }^{89}$ Nach einem Bericht der Sûreté vom 10.7.1935 bildeten die Nationalvolontäre mit 60000 Anhängern die dritte Gruppe nach den eigentlichen Frontkämpfern, den Croix de feu et Briscards mit 100000 und dem Regroupement national mit 80000 Anhängern, APP Ba 1901.

${ }^{90}$ AN F7 13241/3, Berichte vom 11.7. 1935 und 12.7. 1935; B. de Jouvenel, Ce que La Rocque m'a dit, in: Vu, H. 378, 1935, S. 778f.; B. de Jouvenel/Boegner, Scission chez les Croix de Feu, in: Vu, H. 383, 1935, S. 974-976; Burrin, Dérive, S. 190 f.

${ }^{11}$ Mounier, CEuvres, Bd. 4, S. 576.

92 Homme Nouveau, H. 17, 1935, S. 3; Burrin, Dérive, S. $193 f$.
} 
von Aron, der die Ideen des Ordre Nouveau als Basis einer Sammlungsbewegung vorstellte ${ }^{93}$.

In der Zeitschrift Ordre Nouveau setzte sich Rougemont für das Projekt ein. Er sah in den Äußerungen der abtrünnigen Nationalvolontäre ebenso wie in den jüngsten Bekundungen Bergerys und Doriots konstruktive Ansätze für eine Zusammenarbeit. Alle diese Bewegungen verurteilten seiner Ansicht nach den Stalinismus ebenso wie die Diktaturen Mussolinis und Hitlers. Gemeinsam suchten sie einen spezifisch französischen Weg zur Überwindung des liberalen Systems. Rougemont machte jedoch zur Bedingung einer gemeinsamen Aktion, daß sich die Bewegungen den personalistischen Ideen verschrieben. „Es ist Zeit, diesen Truppen eine gemeinsame konstruktive Richtung zu geben, ein Programm, das sie den Unternehmungen des Herrn Wendel ${ }^{94}$ ebenso wie denen des Herrn Litwinow ${ }^{95}$ entgegensetzen können. Auf diese Weise läßt sich die personalistische Lehre in die Tat umsetzen, und die französische Jugend geht Europa mit gutem Beispiel voran."

In diese Gruppe wollte er auch die sogenannten „Grünhemden“ der Landfront mit einbeziehen. Diese Bewegung war unter der Führung Dorgères (eigentlich Henri d'Hallouin) in den Ruf einer militanten Interessenvertretung der kleinen und mittleren Landwirte gegen den Agrarkapitalismus gekommen. Ungeachtet der rechtsextremen Tendenzen der Landfront betrachtete man beim Ordre Nouveau ihren Einsatz für die Interessen der Landbevölkerung mit großer Sympathie. Rougemont sah in der Aktivität der Front die "Keime eines föderalistischen Bewußtseins“ angelegt. Sogar eine direkte Zusammenarbeit zwischen Landfront und dem Ordre Nouveau kam zustande: Achille Bouxin, der als Vertreter der Front an dem Korporatistenkongreß in Rom teilgenommen hatte, fungierte als Korrespondent für Agrarfragen bei Ordre Nouveau ${ }^{97}$.

${ }^{93}$ Mounier wollte jedoch die Jeune Droite ausschließen, die nach seiner Ansicht zu sehr in das Fahrwasser der alten wirtschaftsliberalen Rechten geraten war; Mounier, Faisons le point, in: Esprit, H. 38, 1935, S. 275-281; Aron, Le point de vue de l'Ordre Nouveau, ebenda, S. 281-289; Loustau, Les raisons d'une rupture, ebenda, S. 289-293; Entretiens VIII vom 24. 10. 1935, Mounier, CEuvres, Bd. 4, S. 576.

${ }^{94}$ François de Wendel, Präsident des Comité des Forges, im Aufsichtsrat der Banque de France, Abgeordneter der Fédération républicaine, finanzierte die Jeunesses Patriotes und unterhielt enge Beziehungen zu La Rocque und Doriot; vgl. Jeanney, François de Wendel, S. 485f., 564, $567 \mathrm{ff}$.

${ }^{95}$ Litwinow, eigentlich Maksim Maksimowitsch Wallach, sowjetischer Volkskommissar für Auswärtige Angelegenheiten.

96 Rougemont, Où en est la France?, in: ON, H. 24, 1935, S. 7.

${ }^{97}$ Ebenda; A/chille] B/ouxin], Action paysanne, in: Bulletin ON, Nr. 1, 15. 4. 1935, S. 2; Scrutator [Marc], Les sept jours, in: Sept, H. 59, 1935, S. 2. Die Landfront war im Juli 1934 aus dem Zusammenschluß einer Reihe von Agrarbewegungen hervorgegangen: der Entente paysanne du SudOuest von Edmond Jacquet, Berufsverbänden und Interessenvertretungen wie dem Parti Agraire und der Défense Paysanne von Dorgères. Zu den Delegierten der Landfront, die später zum Front National Paysan umgetauft wurde, gehörte auch Dorgères, der in verschiedenen Kreisen der extremen Rechten aktiv war und Anfang 1936 beim Comité National de Vigilance de la Jeunesse contre la guerre auftauchte; AN F7 13 983, 14, Bericht vom Mai 1936. In der Bewertung der Bewegung sind die Fronten diesmal vertauscht. Soucy betont, die Landfront habe keinen ausgesprochen faschistischen Charakter entwickelt. Ory und Machefer hingegen sehen in dem diktatorischen Programm und in der beabsichtigten Massenmobilisierung durchaus Merkmale einer faschistischen Bewegung; Soucy, Second Wave, S. 43ff.; Machefer, Ligues, S. 28ff.; Ory, Le Dorgérisme, S. 168-190, bes. S. 185 f. 
Marc und Daniel-Rops unterstützten die Sammlungsbewegung von der Zeitschrift Sept aus. Sie gehörten zu den regelmäßigen Mitarbeitern des von den Dominikanern herausgegebenen Blattes und versuchten auf diese Weise, die Katholiken für das Projekt zu gewinnen. Den gemeinsamen Ansatzpunkt dieser Bestrebungen sah Marc in der Suche nach einer neuen Wirtschaftsordnung, die planwirtschaftliche, aber keine diktatorischen Züge trug. Er befürwortete insbesondere den Plan der dissidenten Nationalvolontäre, der seiner Ansicht nach den Prinzipien der katholischen Soziallehre vollkommen entsprach, und rief dazu auf, die ideologischen Gräben mit dem Blick auf das gemeinsame Ziel zu überwinden, „um ihrem Land die Vorstellung von Größe, Gerechtigkeit und Wohltätigkeit zurückzugeben"98. Daniel-Rops betonte die gemeinsame Aufgabe in der Suche nach sozialer Gerechtigkeit, die nur mit dem Umsturz des parlamentarischen Systems verwirklicht werden konnte. Besonders begrüßte er die Abkehr Jacques Doriots vom Kommunismus und dessen Besinnung auf die nationalen Traditionen des Sozialismus. Er ermahnte ihn allerdings, zum Zeichen seiner Aufrichtigkeit auch die Feindschaft zum Christentum zu begraben"9.

Trotz dieser Bemühungen kam die Gemeinschaftsfront nicht zustande. Die Verständigungsversuche endeten im Winter 1935 in einer Sackgasse, ohne daß es allerdings zum offenen Bruch gekommen wäre. Dennoch blieb das Unternehmen nicht ohne Folgen: prominente Verfechter der Sammlungsidee wie die ehemaligen Radikalen Pierre Drieu la Rochelle und Bertrand de Jouvenel schlossen sich der Doriot-Bewegung an, ebenso zahlreiche dissidente Nationalvolontäre, die dem Beispiel ihres Anführers Maud'hui folgten. Unter ihnen war auch Robert Loustau, der innerhalb kurzer Zeit zum wichtigen Theoretiker der Bewegung aufstieg und einen Sitz im Zentralkomitee des Parti Populaire Français (PPF) innehatte, den Doriot 1936 gegründet hatte. Im November 1936 verkündete er auf dem Nationalkongreß des PPF in Saint-Denis die programmatischen Leitsätze der Sozial- und Wirtschaftspolitik der Partei ${ }^{100}$.

Weiterhin nahmen die Hauptakteure des Rassemblement, Bergery, Déat und Doriot, bis zu den Wahlen der Volksfront im Mai 1936 sehr ähnliche Haltungen ein: in dem Urteil über die Volksfront, in ihrer Haltung gegenüber den Sanktionen gegen Italien und der Bewertung der Remilitarisierung des Rheinlands ${ }^{101}$. Welchen Standpunkt der Ordre Nouveau in diesen Fragen einnahm, wird im folgenden Kapitel zu untersuchen sein. Festzuhalten bleibt, daß sowohl der Versuch, eine Sammlungsbewegung auf breiter Basis zu organisieren, als auch sein Scheitern Vorgänge von großer Aussagekraft für die politische Situation Mitte der dreißiger Jahre waren. Sie zeugen von dem Bemühen einer wachsenden Zahl von Dissidenten, die blockierte Modernisierung durch wirtschaftspolitische Strukturreformen zu überwinden und eine neue „Synthese“ zu formulieren. Ihr Scheitern lag

${ }^{88}$ Scrutator [Marc], Les sept jours, in: Sept, H. 83, 1935, S. 2.

99 Daniel-Rops, Union? Regroupement?, in: Sept, H. 89, 1935, S. 16 (Zitat); ders., Communisme National?, in: Sept, H. 94, 1935, S. 16.

100 APP Ba 1946, Bericht vom 10.11. 1936; vgl. seine Artikel in der Parteizeitung Emancipation Nationale seit April 1937; dort hat Loustau sein für den Ordre Nouveau entwickeltes Wirtschaftsund Sozialprogramm vorgestellt, das der neuen Mittelschicht aus Angestellten und Technikern eine vermittelnde Rolle bei der Schlichtung von Konflikten zwischen Arbeitgebern und Arbeitnehmern zuwies, vgl. Wolf, Doriot, S. $123 \mathrm{f}$.

101 Burrin, Dérive, S. 196. 
zu einem großen Teil in dem Unvermögen der Bewegungen begründet, sich auf eine einheitliche Strategie zu einigen. Schon an der Frage, ob die Synthese auf der Grundlage der parlamentarischen Demokratie basieren sollte oder nicht, schieden sich die Geister. Déat, Doriot und Bergery glaubten, auf dem Weg der Reform ihr Ziel erreichen zu können, während für die Nationalvolontäre und den Ordre Nouveau ein Umbau nicht möglich war, ohne gleichzeitig die Prinzipien des Parlamentarismus mit in Frage zu stellen ${ }^{102}$. Der Erfolg der Volksfront machte deutlich, daß eine Sammlungspolitik der Linksparteien im Zeichen des „Antifaschismus“ zu diesem Zeitpunkt mehr überzeugte.

Die Jeune Droite stand diesen Bestrebungen von Anfang an distanziert gegenüber. Selbst Maulnier, der gewisse Sympathien für den „Plan des 9. Juli“ geäußert hatte, sah in der Planwirtschaft nur einen vergeblichen Versuch, Kapitalismus und Sozialismus zu versöhnen. In einer ausführlichen Auseinandersetzung mit den Thesen Hendrik de Mans nach dem Erscheinen der Idée socialiste 1935 formulierte er seine grundsätzlichen Bedenken gegen die Planwirtschaft. Er glaubte, daß mit wirtschaftlichen Strukturreformen nur ein fauler Kompromiß erreicht, keinesfalls aber das liberale System selbst überwunden werden könne. Die ganze Debatte wurde seiner Ansicht nach auf der falschen Ebene geführt. Sie zementierte den Primat der Wirtschaft über die Politik und damit den Sieg der liberalen Bourgeoisie über das Gemeinwohl. Nur in einer radikalen Umkehr, das heißt in der politischen Entmachtung der Bourgeoisie und der Unterwerfung der wirtschaftlichen Interessen unter den Primat der Politik, konnte Maulnier zufolge die Ordnung wiederhergestellt werden ${ }^{103}$.

Diese Argumentation liest sich wie eine Variation des alten Leitmotivs der Action française: „zuerst die Politik“. Aber dies sollte nicht darüber hinwegtäuschen, daß sich die Jeune Droite mittlerweile von den traditionellen Themen der Bewegung deutlich entfernt hatte. Eine charakteristische Verschiebung kennzeichnet die Phase seit 1934: Der Maßstab nationaler Größe war nicht mehr die Außenpolitik. An ihrer Stelle rückte die Frage nach einer stabilen Sozialordnung ins Zentrum des Interesses ${ }^{104}$. Die Versöhnung von Mittelschichten und Arbeitern im Zeichen des "Antikapitalismus" war die Voraussetzung zur Wiederherstellung der nationalen Einheit. Revisionistische Linke und Rechte bemühten sich von verschiedenen Seiten um dasselbe Ziel, hier trafen sich „Planismus“- und Korporatismus-Debatten. Auf der Suche nach dem sozialen Konsens war die Jeune Droite dem gegnerischen Lager auf halbem Weg entgegengekommen.

\section{Zusammenfassung}

Die Februarereignisse des Jahres 1934 gaben in Frankreich das Signal zum Aufbruch. Für die jungen Intellektuellen schien die Zeit reif für einen Umsturz im eigenen Land. Mit wachsender Aufmerksamkeit beobachteten sie die innere Entwicklung des Landes

102 Die grundsätzlich antidemokratische Haltung von La Rocque hat Soucy überzeugend belegt. Dies gilt gleichermaßen für die paramilitärisch organisierten Nationalvolontäre; Soucy, Second Wave, S. $158 \mathrm{ff} ., 109 \mathrm{f}$.

${ }^{103}$ Maulnier, Mythes socialistes, S. 167ff.; ders., Le plan du 9 juillet, in: Mil neuf cent trente quatre,

H. 46, 1934, S. 2; ders., Faiseurs de Plans, in: Courrier Royal, H. 16, 1935, S. 5.

104 Vgl. Hoffmann, Decline or Renewal, S. $418 f$. 
und schmiedeten Pläne für einen Staatsstreich. Keineswegs läßt sich die These von einer Isolation der Gruppierungen aufrechterhalten. Sicherlich markierten die Februarereignisse eine deutliche Zäsur. Fortan stand die Auseinandersetzung um den französischen Faschismus und die Sammlungsbewegung der Linken im Zentrum des Interesses. Doch bedeutete dies nicht das Ende der nonkonformistischen Aufbruchsversuche. Im Gegenteil: Die bis dahin sehr allgemeine und globale Zivilisationskritik erhielt auf einmal konkrete Nahrung. Der Aufmarsch auf der Place de la Concorde hatte deutlich gemacht, daß sich Menschen für Umsturzpläne mobilisieren ließen. Dazu mußten sie allerdings sehr viel konkreter formuliert werden. Und es bedurfte weitreichender Allianzen, um die nötige Schlagkraft zu erlangen. Noch mehr taktische Kompromisse als bisher waren nötig.

Bei der Formulierung der konkreten Staatsentwürfe traten die unterschiedlichen politischen Milieus deutlich zutage. Die Jeune Droite plädierte für einen hierarchischen Staatsaufbau mit starker Exekutive auf der Basis eines Korporativsystems. Die Errungenschaften des Wohlfahrtsstaates wie die Sozialversicherungen sollten soweit wie möglich wieder in privatwirtschaftliche Hände übergehen. Insgesamt handelte es sich um ein wirtschaftsliberales Programm mit korporativen Elementen und einer autoritären Staatsverfassung. Umgekehrt der Ansatz des Ordre Nouveau: er zielte auf die vollständige Überwindung der Legislativorgane und des traditionellen Verwaltungsapparates. Die Gesellschaft sollte sich zukünftig auf kommunaler und regionaler Ebene selbst organisieren. In der Wirtschaftspolitik hingegen sollte der Staat zentrale Kompetenzen besitzen. Mit dem Arbeitsdienst existierte zudem ein Mittel, das Plansoll in zentralen Wirtschaftssektoren zu erfüllen und jedem Bürger eine soziale Absicherung zu garantieren. Eine scheinbare Freiheit im politischen Bereich stand einer starken Reglementierung des wirtschaftlichen Lebens gegenüber. Immerhin gab es sehr fortschrittliche Ansätze wie die Mitbeteiligung der Arbeitnehmer am Produktivvermögen des Unternehmens. Doch wie frei konnte ein Gemeinwesen sein, in dem es außer auf kommunaler Ebene keine demokratischen Wahlen gab, in dem ein zentrales Gremium über die Einhaltung der Staatsziele wachte und diese notfalls direkt durchsetzen konnte?

So unterschiedlich die beiden Entwürfe waren, gab es doch charakteristische Übereinstimmungen. In beiden Fällen war die oberste Staatsführung nicht demokratisch legitimiert. Dezentrale oder föderale Strukturen milderten zwar den Zugriff der Zentralgewalt, doch blieb das ganze System auf eine selbsternannte Elite zugeschnitten. Beide Entwürfe propagierten die Versöhnung von Mittelschichten und Arbeitern im Zeichen des Antikapitalismus. Das Ziel war die Überwindung der Klassengegensätze und die Wiederherstellung der nationalen Einheit. Nach dem Zerfall der „republikanischen Synthese" $z$ wischen Mittelschichten und Bourgeoisie suchten sie nach einer neuen Basis für den gesellschaftlichen und politischen Konsens. Dieser bestand in der Versöhnung von Arbeitern und Mittelschichten. Sie richteten sich ausdrücklich gegen das liberale Bürgertum, das nach Ansicht der Nonkonformisten jegliche Reformen blockiert hatte. Ausgelöst wurde diese Entwicklung durch den Richtungswechsel der Kommunisten, die in der Öffentlichkeit den Klassenkampfparolen abgeschworen hatten. Das Beispiel machte Schule in den zahlreichen Sammlungsbewegungen, die sich als Alternative zur Volksfront formierten. Beide Gruppierungen näherten sich dieser Thematik von unterschiedlichen Seiten. Die Jeune Droite untermauerte ihren Nationalismus deutlich stärker mit sozial- und wirtschaftspolitischen Argumenten. Auf der anderen Seite gerieten die plan- 
wirtschaftlichen Überlegungen des Ordre Nouveau immer deutlicher unter den Primat der Wiederherstellung der nationalen Einheit. Die Pläne einer internationalen Verständigung traten dementsprechend in den Hintergrund.

In jedem Fall setzten die beiden Gruppierungen ihre Strategie einer „Dritten Front" fort und suchten neue Verbündete. Wesentlich stärker als bisher bekannt war, engagierten sich die Anhänger der Jeune Droite und des Ordre Nouveau in den Kreisen extremistischer Ligen und Sammlungsbewegungen. Sie zögerten nicht, mit Bewegungen zu kooperieren, die in der zeitgenössischen Debatte wie auch in der späteren Geschichtsschreibung als faschistisch bezeichnet wurden. 